\title{
GELFAND-FUCHS COHOMOLOGY OF INVARIANT FORMAL VECTOR FIELDS
}

\author{
Ilya Shapiro And Xiang TAng
}

\begin{abstract}
Let $\Gamma$ be a finite group acting linearly on a vector space $V$. We compute the Lie algebra cohomology of the Lie algebra of $\Gamma$-invariant formal vector fields on $V$. We use this computation to define characteristic classes for foliations on orbifolds.
\end{abstract}

\section{Introduction}

The space of smooth vector fields on a manifold $M$ is naturally an infinite dimensional Lie algebra with the usual commutator bracket. The continuous cohomology of this infinite dimensional Lie algebra was studied by Gelfand-Fuchs ([Fu] and references therein). It is now called the Gelfand-Fuchs cohomology of $M$. The study of this cohomology leads to a better understanding of the manifold $M$, in particular, it was proved by Bott-Segal and Haefliger-Trauber that the Gelfand-Fuchs cohomology of $M$ is a homotopy invariant of $M$ and is equal to the singular cohomology of a space functorially constructed from $M$.

The Gelfand-Fuchs cohomology turned out to be a very useful tool in the study of foliations. Berstein-Rozenfeld and Gelfand-Fuchs [Fu] applied it to the construction of the secondary characteristic classes of foliations generalizing the Godbillon-Vey class. Connes and Connes-Moscovici, $[\mathrm{C}]$ and $[\mathrm{CM}]$, adopted the Gelfand-Fuchs cohomology to the study of the transverse index theory for foliations; a Hopf algebraic generalization of the Gelfand-Fuchs cohomology was developed by them.

The connection between the Gelfand-Fuchs cohomology and the algebraic index theory of formal deformation quantizations of symplectic manifolds was developed by Nest-Tsygan $[\mathrm{NT}]$ and Feigin-Felder-Shoikhet [FFS]. The present paper is motivated in part by the second author's study of the algebraic index theory of orbifolds [PPT]. An equivariant version of the Gelfand-Fuchs computation of the cohomology is a crucial step.

Let $V$ be a vector space over $\mathbb{R}$ or $\mathbb{C}$, and $\Gamma$ a finite group acting linearly on $V$. Let $W_{\rho}^{\Gamma}$ be the space of $\Gamma$-invariant formal vector fields on $V$, where $\rho$ denotes the $\Gamma$ action on $V$. We compute the Lie algebra cohomology of $W_{\rho}^{\Gamma}$ as well as some of the relative cases.

In $[\mathrm{PPT}]$ a very special case of the above question was examined. Namely, some nonzero elements in $H^{\bullet}\left(W_{\rho}^{\Gamma}, \mathfrak{g l}(V)^{\Gamma}\right)$ are shown to exist when $V$ is a complex vector space $\left(\mathfrak{g l}(V)^{\Gamma}\right.$ consists of $\Gamma$-invariant linear transformations on $\left.V\right)$. In this paper, we give a much more complete answer to the above question when $V$ is complex, or when $V$ is real and $\Gamma$ is cyclic (with a technical assumption $\Gamma$ may be other than

Received by the editors August 21, 2006. Revision received July 30, 2007. 
cyclic, but the cyclic case is sufficient for an application to the characteristic classes). The methods we use are similar to those of Gelfand-Fuchs [Fu], i.e. Hochschild-Serre spectral sequence and invariant theory. However, we need to deal with a somewhat more complicated algebras and $E_{2}$-terms. Our result compares the Lie algebra cohomology to the cohomology of a certain truncated Weil algebra as well as to the cohomology of a certain topological space naturally associated to a classifying space. This description of the result mirrors closely that of Gelfand-Fuchs. We remark that our computation does depend on the field we work with. The endomorphism algebra of an irreducible representation of $\Gamma$ is $\mathbb{C}$ when $V$ is complex, but may be $\mathbb{R}, \mathbb{C}$, or $\mathbb{H}$ when $V$ is real. We assume $\Gamma$ to be cyclic to avoid the quaternionic case.

As an application, we follow Kontsevich's take on the Gelfand-Fuchs' method [FU, $\mathrm{K}]$ to define characteristic classes for equivariant foliations. When a manifold is equipped with an action of a finite group $\Gamma$, and a foliation $\mathcal{F}$ on $M$ is $\Gamma$-equivariant, $\mathcal{F}$ descends naturally to a foliation on the quotient space $M / \Gamma$. When $M / \Gamma$ is not a manifold but an orbifold, we obtain a foliation $\tilde{\mathcal{F}}$ on the orbifold $M / \Gamma$. We prove that a foliation on an orbifold $X=M / \Gamma$ actually induces a foliation on the corresponding inertia orbifold $\tilde{X}=\coprod_{\langle\gamma\rangle \subset \Gamma} M^{\gamma} / \Gamma^{\gamma}$, where $\langle\gamma\rangle$ is the conjugacy class ${ }^{1}$ of $\gamma$ in $\Gamma, M^{\gamma}$ the fixed point submanifold ${ }^{2}$ of $M$, and $\Gamma^{\gamma}$ consists of fixed points of $\gamma$ in $\Gamma$ under the conjugation action. Though we work always with $M / \Gamma$ our results hold generally for arbitrary orbifolds $X$. Finally, we are able to define characteristic classes for a foliation on an orbifold $X$ as elements in the de Rham cohomology of its inertia orbifold $\tilde{X}$.

The paper is arranged as follows. In Section 2, we compute the Lie algebra cohomology of $W^{\Gamma}$. In Section 3, we prove that a foliation on an orbifold defines a foliation on the corresponding inertia orbifold; applying our computations of the Gelfand-Fuchs cohomology, we define characteristic classes for foliations on orbifolds.

\section{Cohomology of invariant subalgebras of formal vector fields}

Let $V$ be a vector space over $\mathbb{R}$ or $\mathbb{C}$, and $W_{V}$ be the Lie algebra of formal vector fields on $V$. Suppose we have an action $\rho$ of a finite group $\Gamma$ on $V$, this induces an action on $W_{V}$. We compute in this section the Lie algebra cohomology of the Lie algebra $W_{\rho}^{\Gamma}$ of $\Gamma$-invariant formal vector fields on $V$ when $V$ is a complex vector space, or when $V$ is a real vector space and $\Gamma$ is a cyclic group. We divide the computations into several steps. In Section 2.1-2.3, we work out the case of a complex vector space $V$, and in Section 2.4, we explain how to extend our computation to the case of a real vector space $V$ and a cyclic group $\Gamma$ action.

2.1. Eigenvalues of the Euler field. We point out that the $\Gamma$ action on $V$ can be made unitary ${ }^{3}$. Accordingly, as unitary representations of $\Gamma$ are completely reducible,

\footnotetext{
${ }^{1}$ This notation is a possible source of confusion as it is also used to denote the cyclic group generated by $\gamma$, however its meaning is clear from the context.

${ }^{2}$ Note that $M^{\gamma}$ may have quite different connected components.

${ }^{3} \mathrm{Had} \Gamma$ not been a finite or more generally compact group, the condition that the action be unitary would have to be required.
} 
$V$ can be split into

$$
V=V_{0} \oplus \bigoplus_{\alpha=1}^{k} m_{\alpha} W_{\alpha},
$$

where $V_{0}$ is the trivial $\Gamma$ representation, $W_{\alpha}$ is irreducible, and $m_{\alpha}$ is the multiplicity of $W_{\alpha}$ in $V$.

We introduce coordinates on $V$ as follows: $x^{i}$ on $V_{0}, x_{\alpha, s}^{j}$ on $W_{\alpha, s}$ for all $1 \leq \alpha \leq$ $k, 1 \leq s \leq m_{\alpha}, 1 \leq j \leq \operatorname{dim}\left(W_{\alpha}\right)$. We consider the following vector field

$$
\sum_{i, \alpha, s} x_{\alpha, s}^{i} \frac{\partial}{\partial x_{\alpha, s}^{i}}
$$

that we denote by $X$. It is not difficult to see that $X$ is $\Gamma$ invariant and therefore belongs to $W_{\rho}^{\Gamma}$. We need the following Lemma.

Lemma 2.1. Let $\mathfrak{g}$ be a Lie algebra and $X \in \mathfrak{g}$ such that $a d_{\mathfrak{g}} X$ is diagonal with non-negative eigenvalues. Then the inclusion of $\mathfrak{g}^{X}$ into $\mathfrak{g}$ induces and isomorphism on Lie algebra cohomology.

Proof. That $\mathfrak{g}^{X}$ is a subalgebra follows from the Jacobi identity. Since $X$ acts diagonally on $\mathfrak{g}$ it does so also on $\wedge^{\bullet} \mathfrak{g}^{*}$ and each eigenspace is a subcomplex. Since the action of $X$ on cohomology is trivial, only the 0-eigenspace subcomplex contributes non-trivially to the cohomology. By non-negativity of eigenvalues, the 0-eigenspace subcomplex is exactly $\wedge^{\bullet}\left(\mathfrak{g}^{X}\right)^{*}$.

Corollary 2.2. Let $W_{X}=\left(W_{\rho}^{\Gamma}\right)^{X}$, then

$$
H^{\bullet}\left(W_{\rho}^{\Gamma}\right)=H^{\bullet}\left(W_{X}\right)
$$

It is possible to give an explicit description of $W_{X}$ in terms of the decomposition of $V$ into irreducibles. Namely, $W_{X}$ can be identified with $W_{V_{0}} \ltimes\left(\bigoplus_{m_{\alpha}} \operatorname{Poly}\left(V_{0}\right) \otimes\right.$ $\mathfrak{g l}_{m_{\alpha}}(\mathbb{C})$ ), where $W_{V_{0}}$ is the Lie algebra of formal vector fields on $V_{0}$, and $\operatorname{Poly}\left(V_{0}\right)$ is the algebra of polynomials on $V_{0}$. We remark that $W_{V_{0}}$ acts on $\operatorname{Poly}\left(V_{0}\right)$ naturally, and on $\operatorname{Poly}\left(V_{0}\right) \otimes \mathfrak{g l}_{m_{\alpha}}(\mathbb{C})$ via the first factor. This action defines the Lie bracket between $W_{V_{0}}$ and $\bigoplus_{m_{\alpha}} \operatorname{Poly}\left(V_{0}\right) \otimes \mathfrak{g l}_{m_{\alpha}}(\mathbb{C})$. The bracket on $\operatorname{Poly}\left(V_{0}\right) \otimes \mathfrak{g l}_{m_{\alpha}}(\mathbb{C})$ is extended $\operatorname{Poly}\left(V_{0}\right)$-linearly from that on $\mathfrak{g l}_{m_{\alpha}}(\mathbb{C})$.

2.2. Spectral sequence and invariant theory. In this subsection, we will work with the case when there is only one nonzero $m_{\alpha}$ in the representation $\rho$. Namely, we compute the Lie algebra cohomology of $W_{V_{0}} \ltimes \operatorname{Poly}\left(V_{0}\right) \otimes \mathfrak{g l}(W)$, where $V_{0}, W$ are some vector spaces, and the Lie bracket between $W_{V_{0}}$ and $\operatorname{Poly}\left(V_{0}\right) \otimes \mathfrak{g l}(W)$ is defined by the action of $W_{V_{0}}$ on $\operatorname{Poly}\left(V_{0}\right)$.

We consider the Lie subalgebra $\mathfrak{g l}\left(V_{0}\right) \oplus \mathfrak{g l}(W)$ in $W_{V_{0}} \ltimes \operatorname{Poly}\left(V_{0}\right) \otimes \mathfrak{g l}(W)$, and use the Hochschild-Serre spectral sequence to compute the Lie algebra cohomology.

The $E_{1}$ term of this spectral sequence is as follows:

$$
\begin{aligned}
E_{1}^{p, q} & =H^{q}\left(\mathfrak{g l}\left(V_{0}\right) \oplus \mathfrak{g l}(W), \bigwedge^{p}\left(\frac{W_{V_{0}} \ltimes \operatorname{Poly}\left(V_{0}\right) \otimes \mathfrak{g l}(W)}{\mathfrak{g l}\left(V_{0}\right) \oplus \mathfrak{g l}(W)}\right)^{*}\right) \\
& =H^{q}\left(\mathfrak{g l}\left(V_{0}\right) \oplus \mathfrak{g l}(W), \bigwedge^{p}\left(\frac{\operatorname{Sym} V_{0}^{*} \otimes V_{0} \oplus W^{*} \otimes W \otimes \operatorname{Sym} V_{0}^{*}}{V_{0}^{*} \otimes V_{0} \oplus W^{*} \otimes W}\right)^{*}\right) .
\end{aligned}
$$


Thus it is equal to $H^{\bullet}\left(\mathfrak{g l}\left(V_{0}\right) \oplus \mathfrak{g l}(W), \mathbb{C}\right) \otimes \operatorname{Inv}$, where Inv stands for the following expression:

$$
\left(\grave{\bigwedge} V_{0} \otimes \dot{\bigwedge}\left(\mathrm{Sym}^{\geq 2} V_{0}^{*} \otimes V_{0}\right) \otimes \dot{\bigwedge}\left(W \otimes W^{*} \otimes \mathrm{Sym}^{\geq 1} V_{0}^{*}\right)\right)^{* \mathfrak{g r}\left(V_{0}\right) \oplus \mathfrak{g r}(W)}
$$

Let us say a few words of justification for this manipulation. Suppose that $V$, $V_{i}$ are some complex vector spaces. It is a well known fact that $H^{\bullet}(\mathfrak{g l}(V), M)=$ $H^{\bullet}(\mathfrak{g l}(V)) \otimes M^{\mathfrak{g l}(V)}$ provided that $M$ is a tensor module, i.e. is a submodule of $\otimes V \otimes \otimes V^{*}$. The idea of the proof is to introduce an inner product on $V$ which is used to define the Casimir element $\Delta$ of the universal enveloping algebra. The Casimir element (belonging to the center and being self adjoint) gives a decomposition of $\otimes V \otimes \otimes V^{*}$ into $\operatorname{ker} \Delta \oplus \operatorname{im} \Delta$ as a $\mathfrak{g l}(V)$-module. It is then apparent that the module of $\mathfrak{g l}(V)$-invariants (which is exactly $\operatorname{ker} \Delta$ ) is isolated among these submodules as the only submodule with the trivial infinitesimal character. Thus it is the only one contributing non-trivially to the cohomology (as is clear from the Ext interpretation). These considerations then easily apply also to $M$ itself. Using the above ideas it is immediate that $H^{\bullet}\left(\bigoplus \mathfrak{g l}\left(V_{i}\right), M\right)=H^{\bullet}\left(\bigoplus \mathfrak{g l}\left(V_{i}\right)\right) \otimes M^{\oplus \mathfrak{g l}\left(V_{i}\right)}$ provided that $M \subset$ $\otimes\left(\otimes V_{i} \otimes \otimes V_{i}^{*}\right)$, for $i \in I$.

At this point we need to describe the structure of the invariants Inv as an algebra.

\section{Lemma 2.3.}

$$
\begin{aligned}
\left(\grave{\bigwedge} V_{0}\right. & \left.\otimes \bigwedge\left(S y m^{\geq 2} V_{0}^{*} \otimes V_{0}\right) \otimes \dot{\bigwedge}\left(W \otimes W^{*} \otimes S y m^{\geq 1} V_{0}^{*}\right)\right)^{* \mathfrak{g l}\left(V_{0}\right) \oplus \mathfrak{g l}(W)} \\
& =\sum_{r, s}\left(\bigwedge^{r+s} V_{0} \otimes \bigwedge^{r}\left(S y m^{2} V_{0}^{*} \otimes V_{0}\right) \otimes \bigwedge^{s}\left(W \otimes W^{*} \otimes V_{0}^{*}\right)\right)^{* \mathfrak{g l}\left(V_{0}\right) \oplus \mathfrak{g r}(W)} .
\end{aligned}
$$

Proof. This is proved in Proposition 5.2 [PPT]. Here we mention the ingredients. First of all by $\mathfrak{g l}\left(V_{0}\right)$ invariance, elements in $V_{0}$ have to be paired with elements in $V_{0}^{*}$. That is any homogeneous component of an element will have equal numbers of $V_{0}$ and $V_{0}^{*}$. Next, we observe that one cannot pair $\wedge^{k} V_{0}$ with $\operatorname{Sym}^{k} V_{0}^{*}$ in a $\mathfrak{g l}\left(V_{0}\right)$ invariant way for $k>1$. This is sufficient to prove the Lemma.

Using the $\mathfrak{g l}\left(V_{0}\right) \oplus \mathfrak{g l}(W)$-invariance (c. f. [Fu]), we observe that

$$
\left(\bigwedge^{r+s} V_{0} \otimes \bigwedge^{r}\left(\mathrm{Sym}^{2} V_{0}^{*} \otimes V_{0}\right) \otimes \bigwedge^{s}\left(W \otimes W^{*} \otimes V_{0}^{*}\right)\right)^{* \mathfrak{g l}\left(V_{0}\right) \oplus \mathfrak{g l}(W)}
$$

is spanned by $\psi_{\sigma, \gamma}$ with $\sigma \in \Sigma_{r}, \gamma \in \Sigma_{s}$ and

$$
\begin{aligned}
& \psi_{\sigma, \gamma}\left(v_{1}, \ldots, v_{r+s}, \varphi_{1}, v_{1}^{\prime}, \ldots, \varphi_{r}, v_{r}^{\prime}, \ldots, w_{1}, w_{1}^{*}, v_{1}^{*}, \ldots, w_{s}, w_{s}^{*}, v_{s}^{*}\right) \\
& =\sum_{\alpha, \beta, \delta \in \Sigma_{r+s} \times \Sigma_{r} \times \Sigma_{s}} \operatorname{sgn}(\alpha) \operatorname{sgn}(\beta) \operatorname{sgn}(\delta) \prod_{i=1}^{r} \varphi_{\beta(i)}\left(v_{\beta \sigma(i)}^{\prime}, v_{\alpha(i)}\right) \prod_{j=1}^{s} w_{\delta(j)}^{*}\left(w_{\delta \gamma(j)}\right) v_{\delta(j)}^{*}\left(v_{\alpha(r+j)}\right)
\end{aligned}
$$

where $v_{i}, v_{i}^{\prime} \in V_{0}, \varphi_{i} \in \operatorname{Sym}^{2} V_{0}^{*}, w_{j} \in W, w_{j}^{*} \in W^{*}$, and $v_{k}^{*} \in V_{0}^{*}$. 
We will use the notation $\psi_{\sigma, \gamma}\left(\left(v_{i}\right)_{1}^{r+s},\left(\varphi_{i}, v_{i}^{\prime}\right)_{1}^{r},\left(w_{i}, w_{i}^{*}, v_{i}^{*}\right)_{1}^{s}\right)$ to stand for the above pairing. The way to generate such a formula is to write down an element without any symmetry conditions first, i.e.

$$
\prod_{i=1}^{r} \varphi_{i}\left(v_{\sigma(i)}^{\prime}, v_{\alpha(i)}\right) \prod_{j=1}^{s} w_{j}^{*}\left(w_{\gamma(j)}\right) v_{j}^{*}\left(v_{\alpha(r+j)}\right)
$$

and then anti-symmetrize it. Re-indexing the above summations, we get another form of the same element $\psi_{\sigma, \gamma}$ that is evidently constant on the conjugacy classes of $\sigma$ and $\gamma$ :

$$
\sum_{\alpha, \beta, \delta \in \Sigma_{r+s} \times \Sigma_{r} \times \Sigma_{s}} \operatorname{sgn}(\alpha) \prod_{i=1}^{r} \varphi_{i}\left(v_{\beta \sigma \beta^{-1}(i)}^{\prime}, v_{\alpha(i)}\right) \prod_{j=1}^{s} w_{j}^{*}\left(w_{\delta \gamma \delta^{-1}(j)}\right) v_{j}^{*}\left(v_{\alpha(r+j)}\right) .
$$

In order to better understand $\psi_{\sigma, \gamma}$, we define $\Phi_{\sigma} \in\left(\wedge^{r} V_{0} \otimes \wedge^{r}\left(\mathrm{Sym}^{2} V_{0}^{*} \otimes V_{0}\right)\right)^{* \mathfrak{g l}\left(V_{0}\right)}$ and $\Psi_{\gamma} \in\left(\wedge^{s} V_{0} \otimes \wedge^{s}\left(W \otimes W^{*} \otimes V_{0}^{*}\right)\right)^{* \mathfrak{g l}\left(V_{0}\right) \oplus \mathfrak{g l}(W)}$ by

$$
\begin{aligned}
\Phi_{\sigma}\left(\left(v_{i}\right)_{1}^{r},\left(\varphi_{i}, v_{i}^{\prime}\right)_{1}^{r}\right) & =\sum_{\nu, \beta \in \Sigma_{r}} \operatorname{sgn}(\nu) \prod_{i=1}^{r} \varphi_{i}\left(v_{\beta \sigma \beta^{-1}(i)}^{\prime}, v_{\nu(i)}\right), \\
\Psi_{\gamma}\left(\left(v_{i}\right)_{1}^{s},\left(w_{i}, w_{i}^{*}, v_{i}^{*}\right)_{1}^{s}\right) & =\sum_{\eta, \omega \in \Sigma_{s}} \operatorname{sgn}(\eta) \prod_{j=1}^{s} w_{j}^{*}\left(w_{\omega \gamma \omega^{-1}(j)}\right) v_{j}^{*}\left(v_{\eta(j)}\right) .
\end{aligned}
$$

We then have the following:

\section{Lemma 2.4 .}

$$
\Phi_{\sigma} \cdot \Psi_{\gamma}=\psi_{\sigma, \gamma}
$$

Proof.

$$
\begin{aligned}
& \Phi_{\sigma} \cdot \Psi_{\gamma}\left(\left(v_{i}\right)_{1}^{r+s},\left(\varphi_{i}, v_{i}^{\prime}\right)_{1}^{r},\left(w_{i}, w_{i}^{*}, v_{i}^{*}\right)_{1}^{s}\right) \\
& =\frac{1}{r ! s !} \sum_{\alpha \in \Sigma_{r+s}} \operatorname{sgn}(\alpha) \Phi_{\sigma}\left(\left(v_{\alpha(i)}\right)_{1}^{r},\left(\varphi_{i}, v_{i}^{\prime}\right)_{1}^{r}\right) \Psi_{\gamma}\left(\left(v_{\alpha(r+i)}\right)_{1}^{s},\left(w_{i}, w_{i}^{*}, v_{i}^{*}\right)_{1}^{s}\right) \\
& =\frac{1}{r ! s !} \sum_{\alpha \in \Sigma_{r+s}} \operatorname{sgn}(\alpha) \sum_{\nu, \beta \in \Sigma_{r}} \operatorname{sgn}(\nu) \prod_{i=1}^{r} \varphi_{i}\left(v_{\beta \sigma \beta^{-1}(i)}^{\prime}, v_{\alpha \nu(i)}\right) \\
& \sum_{\eta, \omega \in \Sigma_{s}} \operatorname{sgn}(\eta) \prod_{j=1}^{s} w_{j}^{*}\left(w_{\omega \gamma \omega^{-1}(j)}\right) v_{j}^{*}\left(v_{\alpha(r+\eta(j))}\right) \\
& =\frac{1}{r ! s !} \sum_{\nu, \eta} \operatorname{sgn}(\nu \eta) \sum_{\alpha, \beta, \omega} \operatorname{sgn}(\alpha) \prod_{i=1}^{r} \varphi_{i}\left(v_{\beta \sigma \beta^{-1}(i)}^{\prime}, v_{\alpha \nu(i)}\right) \\
& \prod_{j=1}^{s} w_{j}^{*}\left(w_{\omega \gamma \omega^{-1}(j)}\right) v_{j}^{*}\left(v_{\alpha(r+\eta(j))}\right)
\end{aligned}
$$


after re-indexing

$$
\begin{aligned}
& =\frac{1}{r ! s !} \sum_{\nu, \eta} \sum_{\alpha, \beta, \omega} \operatorname{sgn}(\alpha) \prod_{i=1}^{r} \varphi_{i}\left(v_{\beta \sigma \beta^{-1}(i)}^{\prime}, v_{\alpha(i)}\right) \prod_{j=1}^{s} w_{j}^{*}\left(w_{\omega \gamma \omega^{-1}(j)}\right) v_{j}^{*}\left(v_{\alpha(r+j)}\right) \\
& =\psi_{\sigma, \gamma}\left(\left(v_{i}\right)_{1}^{r+s},\left(\varphi_{i}, v_{i}^{\prime}\right)_{1}^{r},\left(w_{i}, w_{i}^{*}, v_{i}^{*}\right)_{1}^{s}\right) .
\end{aligned}
$$

The element $\Phi_{\sigma}$ obviously depends only on the conjugacy class of $\sigma$. Furthermore, if $\sigma \in \Sigma_{r}$ can be decomposed as $\sigma_{1} \coprod \sigma_{2}$ with $\sigma_{i} \in \Sigma_{r_{i}}$ and $r_{1}+r_{2}=r$, then $\Phi_{\sigma}=\Phi_{\sigma_{1}} \cdot \Phi_{\sigma_{2}}$. This was proved in Theorem 2.1.4 [FU]. Thus

$$
\left(\dot{\bigwedge} V_{0} \otimes \dot{\bigwedge}\left(\operatorname{Sym}^{2} V_{0}^{*} \otimes V_{0}\right)\right)^{* \mathfrak{g l}\left(V_{0}\right)}
$$

is generated by $\Phi_{i} \in\left(\bigwedge^{i} V_{0} \otimes \bigwedge^{i}\left(\mathrm{Sym}^{2} V_{0}^{*} \otimes V_{0}\right)\right)^{* \mathfrak{g r}\left(V_{0}\right)}$ for $i=1, \ldots, \operatorname{dim} V_{0}$, given by the elementary cycles of length $i$.

To deal with $\Psi_{\gamma}$, we consider the map:

$$
\sim \quad:\left(\operatorname{Sym}^{s}\left(W \otimes W^{*}\right)\right)^{* \mathfrak{g l}(W)} \rightarrow\left(\bigwedge^{s} V_{0} \otimes \bigwedge^{s}\left(W \otimes W^{*} \otimes V_{0}^{*}\right)\right)^{* \mathfrak{g l}\left(V_{0}\right) \oplus \mathfrak{g l}(W)}
$$

defined by

$$
\widetilde{\Psi}\left(\left(v_{i}\right)_{1}^{s},\left(w_{i}, w_{i}^{*}, v_{i}^{*}\right)_{1}^{s}\right)=\Omega_{s}\left(\left(v_{i}\right)_{1}^{s},\left(v_{i}^{*}\right)_{1}^{s}\right) \Psi\left(\left(w_{i}, w_{i}^{*}\right)_{1}^{s}\right)
$$

where

$$
\Omega_{s}\left(\left(v_{i}\right)_{1}^{s},\left(v_{i}^{*}\right)_{1}^{s}\right)=\sum_{\alpha \in \Sigma_{s}} \operatorname{sgn}(\alpha) \prod_{i=1}^{s} v_{i}^{*}\left(v_{\alpha(i)}\right)
$$

is the canonical element of $\left(\bigwedge^{s} V_{0} \otimes \bigwedge^{s} V_{0}^{*}\right)^{*} \mathfrak{g r}\left(V_{0}\right)$.

Lemma 2.5. The map $\sim$ is a homomorphism.

Proof. Let $\Psi^{s} \in\left(\operatorname{Sym}^{s}\left(W \otimes W^{*}\right)\right)^{* \mathfrak{g l}(W)}$ and $\Psi^{s^{\prime}} \in\left(\operatorname{Sym}^{s^{\prime}}\left(W \otimes W^{*}\right)\right)^{*} \mathfrak{g l}(W)$. Then

$$
\begin{aligned}
\widetilde{\Psi^{s}} \cdot \widetilde{\Psi^{s^{\prime}}}\left(\left(v_{i}\right)_{1}^{s+s^{\prime}},\left(w_{i}, w_{i}^{*}, v_{i}^{*}\right)_{1}^{s+s^{\prime}}\right) \\
=\frac{1}{(s !)^{2}\left(s^{\prime} !\right)^{2}} \sum_{\alpha, \beta \in \Sigma_{s+s^{\prime}}} \operatorname{sgn}(\alpha \beta) \widetilde{\Psi^{s}}\left(\left(v_{\alpha(i)}\right)_{1}^{s},\left(w_{\beta(i)}, w_{\beta(i)}^{*}, v_{\beta(i)}^{*}\right)_{1}^{s}\right) \\
\\
\left.\quad \widetilde{\Psi^{s^{\prime}}}\left(\left(v_{\alpha(s+i)}\right)\right)_{1}^{s^{\prime}},\left(w_{\beta(s+i)}, w_{\beta(s+i)}^{*}, v_{\beta(s+i)}^{*}\right)_{1}^{s^{\prime}}\right) \\
=\frac{1}{s ! s^{\prime} !} \sum_{\beta} \operatorname{sgn}(\beta) \Psi^{s}\left(\left(w_{\beta(i)}, w_{\beta(i)}^{*}\right)_{1}^{s}\right) \Psi^{s^{\prime}}\left(\left(w_{\beta(s+i)}, w_{\beta(s+i)}^{*}\right)_{1}^{s^{\prime}}\right) \\
\\
\quad \cdot \frac{1}{s ! s^{\prime} !} \sum_{\alpha} \operatorname{sgn}(\alpha) \Omega_{s}\left(\left(v_{\alpha(i)}\right)_{1}^{s},\left(v_{\beta(i)}^{*}\right)_{1}^{s}\right) \Omega_{s^{\prime}}\left(\left(v_{\alpha(s+i)}\right)_{1}^{s^{\prime}},\left(v_{\beta(s+i)}^{*}\right)_{1}^{s^{\prime}}\right) \\
=\Omega_{s+s^{\prime}}\left(\left(v_{i}\right)_{1}^{s+s^{\prime}},\left(v_{i}^{*}\right)_{1}^{s+s^{\prime}}\right) \frac{1}{s ! s^{\prime} !} \sum_{\beta} \Psi^{s}\left(\left(w_{\beta(i)}, w_{\beta(i)}^{*}\right)_{1}^{s}\right) \Psi^{s^{\prime}}\left(\left(w_{\beta(s+i)}, w_{\beta(s+i)}^{*}\right)_{1}^{s^{\prime}}\right) \\
=\widetilde{\Psi^{s} \cdot \Psi^{s^{\prime}}}\left(\left(v_{i}\right)_{1}^{s+s^{\prime}},\left(w_{i}, w_{i}^{*}, v_{i}^{*}\right)_{1}^{s+s^{\prime}}\right)
\end{aligned}
$$


The homomorphism obviously factors through the quotient of the symmetric algebra by the ideal of functions of degree greater than $\operatorname{dim}\left(V_{0}\right)$. The induced map from the quotient is than an isomorphism as it is easy to construct an inverse. Namely, consider the evaluation map on any partial basis of $V_{0}$ (this map is choice independent):

$$
\begin{gathered}
\operatorname{ev~}:\left(\bigwedge^{s} V_{0} \otimes \bigwedge^{s}\left(W \otimes W^{*} \otimes V_{0}^{*}\right)\right)^{* \mathfrak{g l}\left(V_{0}\right) \oplus \mathfrak{g l}(W)} \longrightarrow\left(\operatorname{Sym}^{s}\left(W \otimes W^{*}\right)\right)^{* \mathfrak{g l}(W)} \\
\Psi \mapsto \Psi\left(\left(e_{i}\right)_{1}^{s},\left(-,-, e_{i}^{*}\right)_{1}^{s}\right)
\end{gathered}
$$

Then clearly ev $\circ^{\sim}=\mathrm{Id}$, and to check that $\sim \mathrm{ov}=\mathrm{Id}$ it is sufficient to consider $\Psi_{\gamma}$. Recall that $\Psi_{\gamma}\left(\left(v_{i}\right)_{1}^{s},\left(w_{i}, w_{i}^{*}, v_{i}^{*}\right)_{1}^{s}\right)=\sum_{\eta, \omega \in \Sigma_{s}} \operatorname{sgn}(\eta) \prod_{j=1}^{s} w_{j}^{*}\left(w_{\omega \gamma \omega^{-1}(j)}\right) v_{j}^{*}\left(v_{\eta(j)}\right)$ and consider the element $\Psi \in\left(\operatorname{Sym}^{s}\left(W \otimes W^{*}\right)\right)^{* \mathfrak{g l}(W)}$ defined by $\Psi\left(\left(w_{i}, w_{i}^{*}\right)_{1}^{s}\right)=$ $\sum_{\omega \in \Sigma_{s}} \prod_{j=1}^{s} w_{j}^{*}\left(w_{\omega \gamma \omega^{-1}(j)}\right)$. Then $\Psi_{\gamma}=\widetilde{\Psi}$ and this is enough.

Since the structure of $\left(\operatorname{Sym}^{\bullet}\left(W \otimes W^{*}\right)\right)^{* \mathfrak{g r}(W)}$ is well $\mathrm{known}^{4}$, we obtain the structure of $\left(\bigwedge^{\bullet} V_{0} \otimes \Lambda^{\bullet}\left(W \otimes W^{*} \otimes V_{0}^{*}\right)\right)^{* \mathfrak{g l}\left(V_{0}\right) \oplus \mathfrak{g l}(W)}$ as an algebra. Namely it has generators $\widetilde{\Psi}_{i}$ and relations $\prod \widetilde{\Psi}_{i_{k}}=0$ if $\sum i_{k}>\operatorname{dim}\left(V_{0}\right)$. Note that might mean that some of the generators are themselves 0 . We are now ready for:

Lemma 2.6. Inv is generated by $\Phi_{i}, i=1, \cdots, \operatorname{dim}\left(V_{0}\right)$ of degree $2 i$ and $\widetilde{\Psi}_{j}, j=$ $1, \cdots, \operatorname{dim}(W)$ of degree $2 j$ subject to the relation that the total degree can not exceed $2 \operatorname{dim}\left(V_{0}\right)$.

Proof. The only thing that remains is to show that there are no other relations. Suppose that $\sum_{I, J} a_{I, J} \Phi_{I} \widetilde{\Psi}_{J}=0$, where $I$ and $J$ are multi-indices and $|I|+|J| \leq$ $\operatorname{dim}(V) .{ }^{5}$ We may assume that for all $I$ and $J,|I|=r$ and $|J|=s$, thus we may relabel the sum to $\sum_{[\sigma], J} a_{[\sigma], J} \Phi_{\sigma} \widetilde{\Psi}_{J}=0$ with $[\sigma]$ running over the conjugacy classes of $\Sigma_{r}$. Choosing a partial basis $\left(e_{i}\right)_{1}^{r+s}$ of $V$, and a $\tau \in \Sigma_{r}$, set $\varphi_{e_{i}}=\left(e_{i}^{*}\right)^{2}$ and consider the following calculation:

$$
\begin{aligned}
& \Phi_{\sigma} \widetilde{\Psi}_{J}\left(\left(e_{i}\right)_{1}^{r+s},\left(\varphi_{e_{i}}, e_{\tau^{-1}(i)}\right)_{1}^{r},\left(w_{i}, w_{i}^{*}, e_{r+i}^{*}\right)_{1}^{s}\right) \\
& =\frac{1}{r ! s !} \sum_{\alpha \in \Sigma_{r+s}} \operatorname{sgn}(\alpha) \Phi_{\sigma}\left(\left(e_{\alpha(i)}\right)_{1}^{r},\left(\varphi_{e_{i}}, e_{\tau^{-1}(i)}\right)_{1}^{r}\right) \Omega_{s}\left(\left(e_{\alpha(r+i)}\right)_{1}^{s},\left(e_{r+i}^{*}\right)_{1}^{s}\right) \Psi_{J}\left(\left(w_{i}, w_{i}^{*}\right)_{1}^{s}\right) .
\end{aligned}
$$

\footnotetext{
${ }^{4}$ The generators are $\Psi_{i} \in\left(\operatorname{Sym}^{i}\left(W \otimes W^{*}\right)\right)^{* \mathfrak{g l}(W)}$ for $i=1, \ldots, \operatorname{dim}(W)$ corresponding to the elementary cycles of length $i$, and there are no relations.

${ }^{5} \mathrm{By}|I|$ we mean $\sum i_{k}$ where $I=\left\{i_{1}, \ldots, i_{n}\right\}$.
} 
Note that $\Omega_{s}\left(\left(e_{\alpha(r+i)}\right)_{1}^{s},\left(e_{r+i}^{*}\right)_{1}^{s}\right)=0$ unless $\alpha=\alpha_{1} \coprod \alpha_{2}$ with $\alpha_{1} \in \Sigma_{r}$ and $\alpha_{2} \in \Sigma_{s}$, in which case it equals $\operatorname{sgn}\left(\alpha_{2}\right)$, thus

$$
\begin{aligned}
& =\Psi_{J}\left(\left(w_{i}, w_{i}^{*}\right)_{1}^{s}\right) \frac{1}{r !} \sum_{\beta \in \Sigma_{r}} \operatorname{sgn}(\beta) \Phi_{\sigma}\left(\left(e_{\beta(i)}\right)_{1}^{r},\left(\varphi_{e_{i}}, e_{\tau^{-1}(i)}\right)_{1}^{r}\right) \\
& =\Psi_{J}\left(\left(w_{i}, w_{i}^{*}\right)_{1}^{s}\right) \Phi_{\sigma}\left(\left(e_{i}\right)_{1}^{r},\left(\varphi_{e_{i}}, e_{\tau^{-1}(i)}\right)_{1}^{r}\right)
\end{aligned}
$$

Recalling the definition we see that

$$
\begin{aligned}
\Phi_{\sigma}\left(\left(e_{i}\right)_{1}^{r},\left(\varphi_{e_{i}}, e_{\tau^{-1}(i)}\right)_{1}^{r}\right) & =\sum_{\nu, \beta \in \Sigma_{r}} \operatorname{sgn}(\nu) \prod_{i=1}^{r} \varphi_{e_{i}}\left(e_{\tau^{-1} \beta \sigma \beta^{-1}(i)}, e_{\nu(i)}\right) \\
& = \begin{cases}|\operatorname{Stab}(\sigma)| & \text { if } \sigma \sim \tau \\
0 & \text { else. }\end{cases}
\end{aligned}
$$

Therefore evaluating $\sum_{[\sigma], J} a_{[\sigma], J} \Phi_{\sigma} \widetilde{\Psi}_{J}$ at $\left(e_{i}\right)_{1}^{r+s},\left(\varphi_{e_{i}}, e_{\tau^{-1}(i)}\right)_{1}^{r},\left(-,-, e_{r+i}^{*}\right)_{1}^{s}$ we obtain that $\sum_{J} a_{[\tau], J}|\operatorname{Stab}(\tau)| \Psi_{J}=0$ in $\left(\operatorname{Sym}^{*}\left(W \otimes W^{*}\right)\right)^{* \mathfrak{g l}(W)}$, thus $a_{[\tau], J}=0$ for all $\tau$ and $J$.

Remark 2.7. The above Lemma is actually a description of $H^{\bullet}\left(W_{n}^{\Gamma}, G L_{n}^{\Gamma}\right)$ as an algebra. See also Prop. 2.12

2.3. Weil algebras and classifying spaces. In the next step, we relate the cohomology of the Lie algebra $W_{\rho}^{\Gamma}$ to the cohomology of a truncated Weil algebra as well as to the cohomology of a certain topological space.

We recall the general definition of a Weil algebra. Let $\mathfrak{g}$ be a Lie algebra. Define the Weil algebra $W(\mathfrak{g})$ to be $\wedge^{\bullet} \mathfrak{g}^{*} \otimes S^{\bullet} \mathfrak{g}^{*}$. Introduce a grading on $W(\mathfrak{g})$ by assigning elements from $\wedge^{i} \mathfrak{g}^{*} \otimes S^{j} \mathfrak{g}^{*}$ degree $i+2 j$. The algebra $W(\mathfrak{g})$ is filtered by a decreasing filtration

$$
W(\mathfrak{g})=F^{0} W(\mathfrak{g}) \supset F^{1} W(\mathfrak{g})=F^{2} W(\mathfrak{g}) \supset F^{3} W(\mathfrak{g})=F^{4} W(\mathfrak{g}) \supset \cdots,
$$

with $F^{p} W(\mathfrak{g})=\bigoplus_{2 j \geq p} \wedge^{\bullet} \mathfrak{g}^{*} \otimes S^{j} \mathfrak{g}^{*}$.

A differential $d_{W}$ on $W(\mathfrak{g})$ can be introduced as follows:

$$
\begin{aligned}
& d_{W} \psi\left(g_{1} \wedge \cdots \wedge g_{i} \otimes h_{1} \otimes \cdots h_{j}\right)=\sum_{t=1}^{j} \psi\left(g_{1} \wedge \cdots \wedge g_{i} \wedge h_{t} \otimes h_{1} \otimes \cdots \hat{h}_{t} \otimes \cdots \otimes h_{j}\right) \\
& +\sum_{s=1}^{i} \sum_{t=1}^{j}(-1)^{s-1} \psi\left(\left[g_{s}, h_{t}\right] \wedge g_{1} \wedge \cdots \hat{g}_{s} \cdots \wedge g_{i} \otimes h_{1} \otimes \cdots \hat{h}_{t} \cdots \otimes h_{j}\right) \\
& +\sum_{1 \leq s_{1}<s_{2} \leq i}(-1)^{s_{1}+s_{2}-1} \psi\left(\left[g_{s_{1}}, g_{s_{2}}\right] \wedge g_{1} \wedge \cdots \hat{g}_{s_{1}} \cdots \hat{g}_{s_{2}} \cdots \wedge g_{i} \otimes h_{1} \otimes \cdots \otimes h_{j}\right),
\end{aligned}
$$

and $\left(W(\mathfrak{g}), d_{W}, F^{\bullet}\right)$ forms a filtered differential graded algebra. One computes the $E_{2}$ term of the spectral sequence associated to the filtration:

$$
E_{2}^{p, q}= \begin{cases}0, & p \text { is odd } \\ H^{q}\left(\mathfrak{g} ; S^{p / 2} \mathfrak{g}^{*}\right), & p \text { is even }\end{cases}
$$


We note that $(W(\mathfrak{g}), d)$ is acyclic.

The Weil algebra $W(\mathfrak{g})$ has a lifting property which we will use below. Suppose that $\left(C^{\bullet}, \delta\right)$ is a differential graded algebra. Then any linear map $f$ from $\mathfrak{g}^{*}$ to $C^{1}$ of $\left(C^{\bullet}, \delta\right)$ can be lifted to a homogeneous multiplicative homomorphism $F$ from $\left(W(\mathfrak{g}), d_{W}\right)$ to $\left(C^{\bullet}, \delta\right)$, which agrees with $f$ on $\mathfrak{g}^{*}$. To define such a map $F$, it is sufficient to specify the image of the generators:

$$
F\left(g^{*} \otimes 1\right)=f\left(g^{*}\right), \quad F\left(1 \otimes g^{*}\right)=-\delta\left(f\left(g^{*}\right)\right) .
$$

For the purpose of our computation, we need to consider the truncated Weil algebra $W(\mathfrak{g})_{2 n}=W(\mathfrak{g}) / F_{2 n+1} W(\mathfrak{g})$. The filtration $F^{\bullet}$ descends to a filtration on $W(\mathfrak{g})_{2 n}$, and the $E_{2}$-term of the spectral sequence associated to the inherited filtration is

$$
E_{2}^{p, q}= \begin{cases}H^{q}\left(\mathfrak{g} ; S^{p / 2} \mathfrak{g}^{*}\right), & p \text { is even and } p \leq 2 n, \\ 0, & \text { otherwise. }\end{cases}
$$

We consider the special case of $\mathfrak{g}=\mathfrak{g l}\left(V_{0}\right) \oplus \mathfrak{g l}(W)$. To compute the cohomology of the truncated Weil algebra of $\mathfrak{g l}\left(V_{0}\right) \oplus \mathfrak{g l}(W)$, we need to understand the Lie algebra cohomology of $\mathfrak{g l}\left(V_{0}\right) \oplus \mathfrak{g l}(W)$ with coefficient in $S^{\bullet}\left(\mathfrak{g l} \mathfrak{l}\left(V_{0}\right) \oplus \mathfrak{g l}(W)\right)^{*}$. The cohomology $H^{\bullet}\left(\mathfrak{g l}\left(V_{0}\right) \oplus \mathfrak{g l}(W) ; S^{\bullet}\left(\mathfrak{g l}\left(V_{0}\right) \oplus \mathfrak{g l}(W)\right)^{*}\right)$ is isomorphic to

$$
H^{\bullet}\left(\mathfrak{g l}\left(V_{0}\right) ; S^{\bullet} \mathfrak{g l}\left(V_{0}\right)^{*}\right) \otimes H^{\bullet}\left(\mathfrak{g l} l(W) ; S^{\bullet} \mathfrak{g l}(W)^{*}\right) .
$$

By the Casimir element argument, as is explained in Section 2.2, we see that

$$
\begin{aligned}
& H^{\bullet}\left(\mathfrak{g l} l\left(V_{0}\right) ; S^{\bullet} \mathfrak{g l}\left(V_{0}\right)^{*}\right)=H^{\bullet}\left(\mathfrak{g l}\left(V_{0}\right)\right) \otimes\left(S^{\bullet} \mathfrak{g l}\left(V_{0}\right)^{*}\right)^{\mathfrak{g l} l\left(V_{0}\right),} \\
& H^{\bullet}\left(\mathfrak{g l} l(W) ; S^{\bullet} \mathfrak{g l}(W)^{*}\right)=H^{\bullet}(\mathfrak{g l}(W)) \otimes\left(S^{\bullet} \mathfrak{g l}(W)^{*}\right)^{\mathfrak{g l}(W)} .
\end{aligned}
$$

Furthermore by [Fu] [Theorem 2.1.5], $\left(S^{\bullet} \mathfrak{g l}\left(V_{0}\right)^{*}\right)^{\mathfrak{g l}\left(V_{0}\right)}$ is generated by $\xi_{i}, i=1, \cdots$, $\operatorname{dim}\left(V_{0}\right)$, with $\operatorname{deg}\left(\xi_{i}\right)=2 i$ and

$$
\xi_{i}\left(g_{1}, \cdots, g_{i}\right)=\sum_{\sigma \in \Sigma_{i}} \operatorname{Tr}\left(g_{\sigma(1)} \cdots g_{\sigma(i)}\right),
$$

for $g_{s} \in \mathfrak{g l}\left(V_{0}\right)$. Here we view an element $g$ in $\mathfrak{g l}\left(V_{0}\right)$ as a linear endomorphism on $V_{0}$, and $\operatorname{Tr}$ is the trace functional on linear endomorphisms. The same holds for $\left(S^{\bullet} \mathfrak{g l}(W)^{*}\right)^{\mathfrak{g l}(W)}$. Summarizing, we observe that

$$
\begin{aligned}
& H^{\bullet}\left(\mathfrak{g r}\left(V_{0}\right) \oplus \mathfrak{g r}(W) ; S^{\bullet}\left(\mathfrak{g r}\left(V_{0}\right) \oplus \mathfrak{g r}(W)\right)^{*}\right) \\
= & H^{\bullet}\left(\mathfrak{g r}\left(V_{0}\right)\right) \otimes H^{\bullet}(\mathfrak{g r}(W)) \otimes \mathbb{C}\left[\xi_{1}, \cdots, \xi_{\operatorname{dim}\left(V_{0}\right)}\right] \otimes \mathbb{C}\left[\eta_{1}, \cdots, \eta_{\operatorname{dim}(W)}\right] .
\end{aligned}
$$

Let us compute the Lie algebra cohomology of $W_{V_{0}} \ltimes \operatorname{Poly}\left(V_{0}\right) \otimes \mathfrak{g l}(W)$ using the Weil algebra. Consider the "natural" projection $p r$ from $W_{V_{0}} \ltimes \operatorname{Poly}\left(V_{0}\right) \otimes \mathfrak{g l}(W)$ to $\mathfrak{g l}\left(V_{0}\right) \oplus \mathfrak{g l}(W)$. The dual of this map defines a linear map from $\left(\mathfrak{g l}\left(V_{0}\right) \oplus \mathfrak{g l}(W)\right)^{*}$ to $\left(W_{V_{0}} \ltimes \operatorname{Poly}\left(V_{0}\right) \otimes \mathfrak{g l}(W)\right)^{*}$. By the universal lifting property of the Weil algebra we obtain a map

$$
\chi: W\left(\mathfrak{g l}\left(V_{0}\right) \oplus \mathfrak{g l}(W)\right) \rightarrow \wedge^{\bullet}\left(W_{V_{0}} \ltimes \operatorname{Poly}\left(V_{0}\right) \otimes \mathfrak{g l}(W)\right)^{*} .
$$

We write out $\chi$ explicitly on the generators of $W\left(\mathfrak{g l}\left(V_{0}\right) \oplus \mathfrak{g l}(W)\right)$,

$$
\begin{array}{cl}
\chi\left(g^{*} \otimes 1\right)=g^{*}, & \chi\left(h^{*} \otimes 1\right)=h^{*}, \\
\chi\left(1 \otimes g^{*}\right)=-d g^{*}, & \chi\left(1 \otimes h^{*}\right)=-d h^{*},
\end{array}
$$

for $g \in \mathfrak{g l}\left(V_{0}\right), h \in \mathfrak{g l}(W)$. 
We notice that for $g \in \mathfrak{g l}\left(V_{0}\right), h \in \mathfrak{g l}(W), d g^{*} \in V_{0} \wedge \operatorname{Sym}^{2} V_{0}^{*} \otimes V_{0}$ and $d h^{*} \in$ $V_{0} \wedge V_{0}^{*} \otimes W^{*} \otimes W$. It is easy to see that the $\chi$ image of $F^{2 n+1} W\left(\mathfrak{g l}\left(V_{0}\right) \oplus \mathfrak{g l}(W)\right)$ will be contained in $\wedge^{\geq n+1} V_{0} \otimes \wedge^{\bullet} \ldots$. When $n+1$ is greater than the dimension of $V_{0}$, then the above is automatically zero. Therefore, the map $\chi$ factors through the quotient $F^{2 \operatorname{dim}\left(V_{0}\right)+1} W\left(\mathfrak{g l}\left(V_{0}\right) \oplus \mathfrak{g l}(W)\right)$. Furthermore, observing that both $\chi\left(1 \otimes g^{*}\right)=-d g^{*}$ and $\chi\left(1 \otimes h^{*}\right)=-d h^{*}$ are contained in

$$
\wedge^{2}\left(\frac{\operatorname{Sym} V_{0}^{*} \otimes V_{0} \oplus W^{*} \otimes W \otimes \operatorname{Sym} V_{0}^{*}}{V_{0}^{*} \otimes V_{0} \oplus W^{*} \oplus W}\right)^{*},
$$

and both $\chi\left(g^{*} \otimes 1\right)=g^{*}$ and $\chi\left(h^{*} \otimes 1\right)=h^{*}$ are contained in $\mathfrak{g l}\left(V_{0}\right) \oplus \mathfrak{g l}(W)$, we conclude that the map $\chi$ is compatible with the filtrations on $W\left(\mathfrak{g l}\left(V_{0}\right) \oplus \mathfrak{g r}(W)\right)$ and $\wedge^{\bullet}\left(W_{V_{0}} \ltimes \operatorname{Poly}\left(V_{0}\right) \otimes \mathfrak{g l}(W)\right)^{*}$. We remind the reader that the filtration on $\wedge \bullet\left(W_{V_{0}} \ltimes \operatorname{Poly}\left(V_{0}\right) \otimes \mathfrak{g l}(W)\right)^{*}$ (its associated spectral sequence is the Hochschild-Serre spectral sequence for the Lie subalgebra $\left.\mathfrak{g l}\left(V_{0}\right) \oplus \mathfrak{g l}(W)\right)$ is defined by the powers of the wedge

$$
\wedge \cdot\left(\frac{\operatorname{Sym} V_{0}^{*} \otimes V_{0} \oplus W^{*} \otimes W \otimes \operatorname{Sym}_{0}^{*}}{V_{0}^{*} \otimes V_{0} \oplus W^{*} \otimes W}\right)^{*} .
$$

In conclusion, we have constructed a filtration compatible differential graded algebra morphism

$$
\chi: W\left(\mathfrak{g l}\left(V_{0}\right) \oplus \mathfrak{g l}(W)\right)_{2 \operatorname{dim}\left(V_{0}\right)} \rightarrow \wedge^{\bullet}\left(W_{V_{0}} \ltimes \operatorname{Poly}\left(V_{0}\right) \otimes \mathfrak{g l}(W)\right)^{*},
$$

where $W\left(\mathfrak{g l}\left(V_{0}\right) \oplus \mathfrak{g l}(W)\right)_{2 \operatorname{dim}\left(V_{0}\right)}$ is the truncated Weil algebra.

Lemma 2.8. The map $\chi$ defined above is a quasi-isomorphism.

Proof. Since $\chi$ is compatible with the filtrations, it is sufficient to prove that $\chi$ is an isomorphism on the $E_{2}$ terms of the spectral sequences associated to the filtrations. As we know that $\chi$ is multiplicative, it is sufficient to show that $\chi$ defines isomorphisms on $E_{2}^{p, 0}$ and $E_{2}^{0, q}$.

For $E_{2}^{0, q}$, we easily see from Equation (1) that $\chi$ is an identity map on $\wedge \cdot \mathfrak{g l}\left(V_{0}\right) \otimes$ $\wedge \mathfrak{g l}(W)$.

For $E_{2}^{p, 0}$, we observe that $E_{2}^{\bullet, 0}$ forms an algebra. And as $\chi$ is multiplicative, this reduces to checking the statement on the generators. Observe that $E_{2}^{\bullet, 0}$ of $W\left(\mathfrak{g l}\left(V_{0}\right) \oplus\right.$ $\mathfrak{g l}(W))$ is generated by $\xi_{i}$ and $\eta_{j}, i=1, \cdots, \operatorname{dim}\left(V_{0}\right)$ and $j=1, \cdots \operatorname{dim}(W)$. We compute $\chi\left(\xi_{i}\right)$ and $\chi\left(\eta_{j}\right)$.

(1) The image $\chi\left(\xi_{i}\right)$ was already considered in the proof of Theorem 2.2.4', [Fu]. Hence, we skip the particulars of this part. The conclusion is that up to a nonzero constant the image of $\chi\left(\xi_{i}\right)$ is $\Phi_{i}$.

(2) For $\chi\left(\eta_{j}\right)$, looking at Equation (1), we observe that $\chi\left(\eta_{j}\right)$ is in

$$
\wedge^{j} V_{0} \otimes \wedge^{j}\left(W^{*} \otimes W \otimes V^{*}\right),
$$

as $\chi(1 \otimes h)$ is in $V_{0} \otimes W^{*} \otimes W \otimes V_{0}^{*}$.

Recall that for an element $h \in \mathfrak{g l}(W)^{*}, \chi(h)\left(v, w \otimes w^{*} \otimes v^{\prime}\right)=h([v, w \otimes$ $\left.\left.w^{*} \otimes v^{\prime}\right]\right)=v^{\prime}(v) h\left(w \otimes w^{*}\right)$, for $v \in V_{0}, v^{\prime} \in V^{*}, w \in W$, and $w^{*} \in W^{*}$. As $\chi$ is an algebra homomorphism, we generalize the above evaluation to $\chi\left(\eta_{j}\right)$, 
i.e.

$$
\begin{aligned}
& \chi\left(\eta_{j}\right)\left(v_{1}, \cdots, v_{j}, w_{1}^{*}, w_{1}^{\prime}, v_{1}^{*}, \cdots, w_{j}^{*}, w_{j}^{\prime}, v_{j}^{*}\right) \\
= & \prod_{\sigma \in \Sigma_{j}} \operatorname{sgn}(\sigma) v_{1}^{*}\left(v_{\sigma(1)}\right) \cdots v_{j}^{*}\left(v_{\sigma(j)}\right) \eta_{j}\left(w_{1}^{*} \otimes w_{1}^{\prime}, \cdots, w_{j}^{*} \otimes w_{j}^{\prime}\right) .
\end{aligned}
$$

We recall that $\eta_{j}$ is the element in $\left(\operatorname{Sym}^{j} \mathfrak{g l}(W)^{*}\right)^{\mathfrak{g l}(W)}$ corresponding to the $j$-cycle in the permutation group $\Sigma_{j}$. The second line of the above equation agrees with the $\widetilde{\Psi}_{j}$ 's evaluation on $v_{1}, \cdots, v_{j}, w_{1}^{*}, w_{1}, v_{1}^{*}, \cdots, w_{j}^{*}, w_{j}, v_{j}^{*}$. Therefore, we have that $\chi\left(\eta_{j}\right)=\widetilde{\Psi}_{j}$.

With Lemma 2.6, we conclude that $\chi$ is an isomorphism on the $E_{2}$ terms of the spectral sequences and therefore induces an isomorphism on the cohomologies.

Having dealt with the special case of a single nonzero $m_{\alpha}$, we can state the result in full generality. The proof is identical though more notationally intensive and so we omit it.

Theorem 2.9. Let $\Gamma$ be a finite group acting linearly on a complex vector space $V$. Suppose that $V$ decomposes as $V=V_{0} \oplus \bigoplus_{\alpha=1}^{k} m_{\alpha} W_{\alpha}$ into irreducible representations of $\Gamma$, with $V_{0}=V^{\Gamma}$. Then

$$
H^{\bullet}\left(W_{\rho}^{\Gamma}\right)=H^{\bullet}\left(W\left(\mathfrak{g l}\left(V_{0}\right) \oplus \bigoplus_{\alpha=1}^{k} \mathfrak{g l}_{m_{\alpha}}(\mathbb{C})\right)_{2 \operatorname{dim}\left(V_{0}\right)}\right) .
$$

We now wish to give a topological description of $H^{\bullet}\left(W_{\rho}^{\Gamma}\right)$. Consider the principal bundle

$$
\pi: E\left(G L\left(V_{0}\right) \times \prod_{\alpha=1}^{k} G L_{m_{\alpha}}(\mathbb{C})\right) \rightarrow B\left(G L\left(V_{0}\right) \times \prod_{\alpha=1}^{k} G L_{m_{\alpha}}(\mathbb{C})\right) .
$$

Denote by $B_{\rho}^{\Gamma}$ the $2 \operatorname{dim}\left(V_{0}\right)$-th skeleton of $B\left(G L\left(V_{0}\right) \times \prod_{\alpha=1}^{k} G L_{m_{\alpha}}(\mathbb{C})\right)$, and by $X_{\rho}^{\Gamma}$ its $\pi$ preimage inside $E\left(G L\left(V_{0}\right) \times \prod_{\alpha=1}^{k} G L_{m_{\alpha}}(\mathbb{C})\right)$. The following Theorem is then almost an immediate corollary of Thm. 2.9

Theorem 2.10. $H^{\bullet}\left(W_{\rho}^{\Gamma}\right) \cong H^{\bullet}\left(X_{\rho}^{\Gamma}\right)$.

Proof. The proof is identical to that of Theorem 2.2.4', [Fu]. Here we outline the main ingredients. We compare the spectral sequence associated to the filtration on $W\left(\mathfrak{g l}\left(V_{0}\right) \oplus \bigoplus_{\alpha=1}^{k} \mathfrak{g l}_{m_{\alpha}}(\mathbb{C})\right)_{2 \operatorname{dim}\left(V_{0}\right)}$ with the spectral sequence of the bundle $X_{\rho}^{\Gamma} \rightarrow$ $B_{\rho}^{\Gamma}$. From the previous computation, we have seen that $E_{2}$ terms of the two spectral sequences agree as algebras. Furthermore, by the acyclicity of the untruncated Weil algebra $W\left(\mathfrak{g l}\left(V_{0}\right) \oplus \bigoplus_{\alpha} \mathfrak{g l}_{m_{\alpha}}(\mathbb{C})\right)$ and the cohomology of $E\left(G L\left(V_{0}\right) \times \prod_{\alpha=1}^{k} G L_{m_{\alpha}}(\mathbb{C})\right)$, we see that in the two spectral sequences the exterior generators of the algebra

$$
E_{2}^{0, \bullet}=H^{\bullet}\left(\mathfrak{g l}\left(V_{0}\right) \oplus \bigoplus_{\alpha=1}^{k} \mathfrak{g l}_{m_{\alpha}}(\mathbb{C})\right)=H^{\bullet}\left(G L\left(V_{0}\right) \times \prod_{\alpha=1}^{k} G L_{m_{\alpha}}(\mathbb{C})\right)
$$

are transgressive and mapped to the multiplicative generators of the algebra

$$
E_{2}^{\bullet, 0}=\left(S^{\bullet}\left(\mathfrak{g l}\left(V_{0}\right) \oplus \bigoplus_{\alpha=1}^{k} \mathfrak{g l}_{m_{\alpha}}(\mathbb{C})\right)^{*}\right)_{2 \operatorname{dim}\left(V_{0}\right)}^{\mathfrak{g l}\left(V_{0}\right) \oplus \bigoplus_{\alpha} \mathfrak{g l}_{m_{\alpha}}(\mathbb{C})}=H^{\bullet}\left(B_{\rho}^{\Gamma}\right)
$$


by the transgression. Therefor, we conclude that the limits of the two spectral sequences agree.

Remark 2.11. When both $V$ and $W$ are 1 dimensional, the Lie algebra cohomology of $W_{V} \ltimes \operatorname{Poly}(V) \otimes \mathfrak{g l}(W)$ can be computed directly without using spectral sequence. Let $x$ be the variable on $V$ and $y$ be the variable on $W$. Consider the vector field $x \frac{\partial}{\partial x} \in \mathfrak{g l}(V)$. We find that the adjoint action of $x \frac{\partial}{\partial x}$ on $W_{V} \ltimes \operatorname{Poly}(V) \otimes \mathfrak{g l}(W)$ and therefore on the cochain complex is diagonal. Accordingly, the cohomology of $W_{V} \ltimes \operatorname{Poly}(V) \otimes \mathfrak{g l}(W)$ is computed by the 0 eigenvectors in

$$
\dot{\bigwedge}\left(W_{V} \ltimes \operatorname{Poly}(V) \otimes \mathfrak{g l}(W)\right)^{*} .
$$

We can find these 0 eigenvectors explicitly and compute the Lie algebra cohomology easily without using spectral sequence.

We now have the language to state the following Proposition:

Proposition 2.12. $H^{\bullet}\left(W_{\rho}^{\Gamma}, \mathfrak{g l}\left(V_{0}\right) \oplus \bigoplus_{\alpha=1}^{k} \mathfrak{g l}_{m_{\alpha}}(\mathbb{C})\right)=H^{\bullet}\left(B_{\rho}^{\Gamma}\right)$.

2.4. The real case. In this subsection, we consider the real Lie algebra $W_{\rho}^{\Gamma}$ of $\Gamma$ invariant formal vector fields on a real vector space $V$, where $\rho$ denotes the $\Gamma$ representation. Let us assume that $\Gamma$ is a finite cyclic group as that is the case we need for defining characteristic classes of foliation on an orbifold.

As $\Gamma$ is finite, the $\Gamma$ action on $V$ is completely reducible. Suppose that $U$ is an irreducible component. There are two possibilities: $U \otimes \mathbb{C}$ is irreducible, thus $\operatorname{End}_{\Gamma}(U \otimes \mathbb{C})=\mathbb{C}$ and so $\operatorname{End}_{\Gamma}(V)=\mathbb{R}$ or $U \otimes \mathbb{C}=W \oplus \bar{W}$ with $W$ irreducible in which case $\operatorname{End}_{\Gamma}(U \otimes \mathbb{C})=\mathbb{C}^{2}$ since $^{6} W=\bar{W}$ (they correspond to different eigenvalues of the generator of $\Gamma$ ), and so $\operatorname{End}_{\Gamma}(V)=\mathbb{C}$. Therefore, we can write, similar to the complex case:

$$
V=V_{0} \oplus m_{-1} W_{-1} \oplus \bigoplus_{\alpha=1}^{k} m_{\alpha} W_{\alpha},
$$

where $\Gamma$ acts on $V_{0}$ trivially, $W_{-1}$ is the one dimensional representation with character $-1, W_{\alpha}$ is an irreducible representation of $\Gamma$ with $\operatorname{dim}_{\mathbb{R}}\left(W_{\alpha}\right)=2$, and $m_{\alpha}$ is the multiplicity of the representation $W_{\alpha}$ in $V$.

Similar to the complex case in Section 2.1, there is a $\Gamma$-invariant Euler vector field $X$ on $\mathbb{R}^{n}$, which acts on $W_{V}$ diagonally with nonnegative eigenvalues. As with Corollary 2.2 , by looking at the eigenvalues of $X$ we reduce the Lie algebra $W_{\rho}^{\Gamma}$ to $W_{X}$, where $W_{X}$ consists of eigenvectors of $X$ in $W_{\rho}^{\Gamma}$ with zero eigenvalue. We see that $W_{X}$ is somewhat different from the complex case:

$$
W_{X}=W_{V_{0}} \ltimes \operatorname{Poly}\left(V_{0}\right) \otimes\left(\mathfrak{g l}_{m_{-1}}(\mathbb{R}) \oplus \bigoplus_{\alpha=1}^{k} \boldsymbol{g l}_{m_{\alpha}}\right),
$$

where $\boldsymbol{g l}_{m_{\alpha}}$ is the complex general Lie algebra $\mathfrak{g l}_{m_{\alpha}}(\mathbb{C})$ viewed as a real Lie algebra.

Extending results 2.2-2.8, we have

\footnotetext{
${ }^{6}$ This is where one needs the assumption that $\Gamma$ is cyclic. Alternatively, we may simply assume that $W \neq \bar{W}$. This rules out the quaternions.
} 
Proposition 2.13. There is a natural quasi-isomorphism $\chi$ from the truncated Weil algebra

$$
W\left(\mathfrak{g l}\left(V_{0}\right) \oplus \mathfrak{g l}_{m_{-1}}(\mathbb{R}) \oplus \bigoplus_{\alpha=1}^{k} \boldsymbol{g l}_{m_{\alpha}}\right)_{2 \operatorname{dim}\left(V_{0}\right)}
$$

to the cochain complex of the real Lie algebra $W_{\rho}^{\Gamma}$.

Proof. The proof is a copy of that of Lemma 2.8. The map $\chi$ is defined by the projection from $W_{X}$ to $\mathfrak{g l}\left(V_{0}\right) \oplus \mathfrak{g l}_{m_{-1}}(\mathbb{R}) \oplus \bigoplus_{\alpha=1}^{k} \boldsymbol{g l}_{m_{\alpha}}$. To prove that $\chi$ is a quasiisomorphism, we compare the $E_{2}$ terms of spectral sequences associated to the filtration on the Weil algebra $W\left(\mathfrak{g l}\left(V_{0}\right) \oplus \mathfrak{g l}_{m_{-1}}(\mathbb{R}) \oplus \bigoplus_{\alpha=1}^{k} \boldsymbol{g l}_{m_{\alpha}}\right)_{2 \operatorname{dim}\left(V_{0}\right)}$ and to the Lie subalgebra $\mathfrak{g l}\left(V_{0}\right) \oplus \mathfrak{g l}_{m_{-1}}(\mathbb{R}) \oplus \bigoplus_{\alpha=1}^{k} \boldsymbol{g} \boldsymbol{l}_{m_{\alpha}}$ of $W_{X}$. One change we need to make is to replace $\mathfrak{g l}_{m_{\alpha}}(\mathbb{C})$ by $\boldsymbol{g l}_{m_{\alpha}}$, while the algebra of invariant polynomials on $\boldsymbol{g l}_{m_{\alpha}}$ is computed in Proposition 2.16.

Furthermore, we may choose a $\Gamma$-invariant metric on $V$ and consider the Lie subalgebra $\mathfrak{o}\left(V_{0}\right) \oplus \mathfrak{o}_{m_{-1}}(\mathbb{R}) \oplus \bigoplus_{\alpha=1}^{k} \mathfrak{u}_{m_{\alpha}}$ inside $W_{\rho}^{\Gamma}$, where $\mathfrak{o}$ is the Lie algebra of orthogonal matrices, and $\mathfrak{u}$ is the Lie algebra of skew-hermitian matrices.

We now use the relative version of the Hochschild-Serre spectral sequence to compute the relative cohomology $H^{\bullet}\left(W_{\rho}^{\Gamma}, \mathfrak{o}\left(V_{0}\right) \oplus \mathfrak{o}_{m_{-1}}(\mathbb{R}) \oplus \bigoplus_{\alpha=1}^{k} \mathfrak{u}_{m_{\alpha}}\right)$. We need the following Lemma:

Lemma 2.14. Let $V_{i}$ be real vector spaces, $W_{i}$ complex vector spaces, $\mathfrak{o}_{i}$ and $\mathfrak{u}_{i}$ reductive subalgebras of $\mathfrak{g l}\left(V_{i}\right)$ and $\boldsymbol{g l}\left(W_{i}\right)$ respectively, then

$$
\begin{aligned}
H^{\bullet}\left(\bigoplus \mathfrak{g l}\left(V_{i}\right)\right. & \left.\oplus \bigoplus \boldsymbol{g l}\left(W_{i}\right), \bigoplus \mathfrak{o}_{i} \oplus \bigoplus \mathfrak{u}_{i} ; M\right) \\
& =H^{\bullet}\left(\bigoplus \mathfrak{g l}\left(V_{i}\right) \oplus \bigoplus \boldsymbol{g l}\left(W_{i}\right), \bigoplus \mathfrak{o}_{i} \oplus \bigoplus \mathfrak{u}_{i}\right) \otimes M^{\oplus \mathfrak{g l}\left(V_{i}\right) \oplus \oplus \boldsymbol{g l}\left(W_{i}\right)}
\end{aligned}
$$

provided $M$ is a "tensor" module.

Proof. Observe that $H^{\bullet}(\mathfrak{g}, \mathfrak{k}, M)=H^{\bullet}(\mathfrak{g}, \mathfrak{k}) \otimes M^{\mathfrak{g}}$ if its complexified version holds. Note also that $\boldsymbol{g l}(W) \otimes \mathbb{C}=\mathfrak{g l}_{\mathbb{C}}(W) \oplus \mathfrak{g l}_{\mathbb{C}}(W)$; this decomposition is given by the eigenspaces of the "forgotten" multiplication by $i$ acting on $\boldsymbol{g l}(W)$. The meaning of a "tensor" module is now clear. We may use the Ext interpretation of the relative cohomology (valid for reductive subalgebras, see $[\mathrm{BW}]$ for example) to conclude that only a module with a trivial infinitesimal character contributes and thus the earlier discussion of cohomology of tensor modules over $\bigoplus \mathfrak{g l}\left(V_{i}\right)$ applies.

Proposition 2.15. Let

$$
W\left(\mathfrak{g l}\left(V_{0}\right) \oplus \mathfrak{g l}_{m_{-1}}(\mathbb{R}) \oplus \bigoplus_{\alpha=1}^{k} \boldsymbol{g} \boldsymbol{l}_{m_{\alpha}}, \mathfrak{o}\left(V_{0}\right) \oplus \mathfrak{o}_{m_{-1}}(\mathbb{R}) \oplus \bigoplus_{\alpha=1}^{k} \mathfrak{u}_{m_{\alpha}}\right)_{2 \operatorname{dim}\left(V_{0}\right)}
$$

be the truncated (at degree $>2 \operatorname{dim}\left(V_{0}\right)$ ) relative Weil algebra. The map $\chi$ descends to a quasi-isomorphism to the relative cochain complex $C^{\bullet}\left(W_{\rho}^{\Gamma}, \mathfrak{o}\left(V_{0}\right) \oplus \mathfrak{o}_{m_{-1}}(\mathbb{R}) \oplus\right.$ $\left.\bigoplus_{\alpha=1}^{n} \mathfrak{u}_{m_{\alpha}}\right)$.

Proof. The proof consists of checking that the $\chi$ is a quasi-isomorphism on the $E_{2}$ terms associated to the relative spectral sequences, which is analogous to the proof of Lemma 2.8. 
To obtain a topological description of the cohomology we compute the invariants of $\boldsymbol{g} \boldsymbol{l}_{m}$ as follows.

Proposition 2.16. There is an isomorphism of algebras

$$
\left(S^{\bullet} \boldsymbol{g} \boldsymbol{l}_{m}\right)^{*} \boldsymbol{g} \boldsymbol{l}_{m}=\mathbb{R}\left[x_{s}, y_{s}\right]_{s=1}^{m}
$$

with degree of $x_{s}$ and $y_{s}$ equal to $s$.

Proof. As is explained in the proof of Lemma 2.14, the complexification of $\boldsymbol{g} \boldsymbol{l}_{m}$ is isomorphic to $\mathfrak{g l}_{m} \mathbb{C} \oplus \mathfrak{g l}_{m}(\mathbb{C})$. While $\left(S^{\bullet} \mathfrak{g l}_{m}(\mathbb{C})\right)^{* \mathfrak{g l}_{m}(\mathbb{C})}=\mathbb{C}\left[z_{s}\right]_{s=1}^{m}$ with $z_{s}$ of degree $s$ given by the symmetric trace, and so $\left(S^{\bullet} \boldsymbol{g} \boldsymbol{l}_{m} \otimes \mathbb{C}\right) * \boldsymbol{g} \boldsymbol{l}_{m} \otimes \mathbb{C}$ is a complex polynomial algebra with $2 m$ generators $z_{s}$ and $t_{s}$. It is straight forward to trace through the isomorphisms to check that $\left(S^{\bullet} \boldsymbol{g} \boldsymbol{l}_{m}\right)^{*} \boldsymbol{g} \boldsymbol{l}_{m}$ (as the subalgebra on which conjugation acts trivially) is isomorphic to $\mathbb{R}\left[\frac{z_{s}+t_{s}}{2}, \frac{z_{s}-t_{s}}{2 i}\right]$.

Proposition 2.17. There exist isomorphisms:

(1) $H^{\bullet}\left(\boldsymbol{g l}_{m}\right)=H^{\bullet}\left(U_{m} \times U_{m}\right)$,

(2) $H^{\bullet}\left(\boldsymbol{g l}_{m}, \mathfrak{u}_{m}\right)=H^{\bullet}\left(U_{m}\right)$.

Proof. For statement (1), we notice that $\boldsymbol{g l}_{m} \otimes \mathbb{C}=\mathfrak{g l}_{m}(\mathbb{C}) \oplus \mathfrak{g l}_{m}(\mathbb{C})=\left(\mathfrak{u}_{m} \oplus \mathfrak{u}_{m}\right) \otimes \mathbb{C}$. Therefore, $H^{\bullet}\left(\boldsymbol{g l}_{m}\right)=H^{\bullet}\left(\mathfrak{u}_{m} \oplus \mathfrak{u}_{m}\right)=H^{\bullet}\left(U_{m} \times U_{m}\right)$ because $U_{m} \times U_{m}$ is a compact Lie group.

For statement (2), we observe that under the isomorphism $\boldsymbol{g l}_{m} \otimes \mathbb{C}=\mathfrak{g l}_{m}(\mathbb{C}) \oplus$ $\mathfrak{g l}_{m}(\mathbb{C})$, the complexification of $\mathfrak{u}_{m}$ is identified with $\mathfrak{g l}_{m}(\mathbb{C})$ embedded diagonally into $\boldsymbol{g l}_{m} \otimes \mathbb{C}$. Furthermore, the pair $\left(\mathfrak{g l}_{m}(\mathbb{C}) \oplus \mathfrak{g l}_{m}(\mathbb{C}), \mathfrak{g l}_{m}(\mathbb{C})\right)$ is the complexification of $\left(\mathfrak{u}_{m} \oplus \mathfrak{u}_{m}, \mathfrak{u}_{m}\right)$ with $\mathfrak{u}_{m}$ embedded into $\mathfrak{u}_{m} \oplus \mathfrak{u}_{m}$ diagonally. Therefore, $H^{\bullet}\left(\boldsymbol{g} \boldsymbol{l}_{m}, \mathfrak{u}_{m}\right)=$ $H^{\bullet}\left(\mathfrak{u}_{m} \oplus \mathfrak{u}_{m}, \mathfrak{u}_{m}\right)=H^{\bullet}\left(U_{m} \times U_{m} / U_{m}\right)=H^{\bullet}\left(U_{m}\right)$.

By Proposition 2.16, $\left(S^{\bullet} \boldsymbol{g} \boldsymbol{l}_{m}\right)^{*} \boldsymbol{g} \boldsymbol{l}_{m}$ is isomorphic to the cohomology ring of the classifying space $B\left(U_{m} \times U_{m}\right)$, a polynomial ring with $2 m$ generators. This brings to mind the fibration $E\left(U_{m} \times U_{m}\right) \rightarrow B\left(U_{m} \times U_{m}\right)$ with the fiber $U_{m} \times U_{m}$. Thus we obtain the following topological description of the cohomology computed in Proposition 2.13 and 2.15. Consider the fibration

$$
\begin{aligned}
\pi: E\left(U_{\operatorname{dim}\left(V_{0}\right)} \times U_{m_{-1}} \times\right. & \left.\prod_{\alpha=1}^{k}\left(U_{m_{\alpha}} \times U_{m_{\alpha}}\right)\right) \\
& \longrightarrow B\left(U_{\operatorname{dim}\left(V_{0}\right)} \times U_{m_{-1}} \times \prod_{\alpha=1}^{k}\left(U_{m_{\alpha}} \times U_{m_{\alpha}}\right)\right) .
\end{aligned}
$$

Let $X_{\rho}^{\Gamma}$ be the $\pi$ preimage in $E\left(U_{\operatorname{dim}\left(V_{0}\right)} \times U_{m_{-1}} \times \prod_{\alpha=1}^{k}\left(U_{m_{\alpha}} \times U_{m_{\alpha}}\right)\right)$ of the $2 \operatorname{dim}\left(V_{0}\right)$ th skeleton $B_{\rho}^{\Gamma}$ of $B\left(U_{\operatorname{dim}\left(V_{0}\right)} \times U_{m_{-1}} \times \prod_{\alpha=1}^{k}\left(U_{m_{\alpha}} \times U_{m_{\alpha}}\right)\right)$. Note that $S O\left(V_{0}\right) \times$ $S O_{m_{-1}}(\mathbb{R}) \times \prod_{\alpha=1}^{k} U_{m_{\alpha}}$ and $O\left(V_{0}\right) \times O_{m_{-1}}(\mathbb{R}) \times \prod_{\alpha=1}^{k} U_{m_{\alpha}}$ act on the fibers of $X_{\rho}^{\Gamma} \rightarrow$ $B_{\rho}^{\Gamma}\left(U_{m_{\alpha}}\right.$ 's are embedded diagonally in $\left.U_{m_{\alpha}} \times U_{m_{\alpha}}\right)$. We then have:

Theorem 2.18.

$$
\begin{gathered}
H^{\bullet}\left(W_{\rho}^{\Gamma}\right)=H^{\bullet}\left(X_{\rho}^{\Gamma}\right) \\
H^{\bullet}\left(W_{\rho}^{\Gamma}, \mathfrak{g l}\left(V_{0}\right) \oplus \mathfrak{g l}_{m_{-1}}(\mathbb{R}) \oplus \bigoplus_{\alpha=1}^{k} \boldsymbol{g} \boldsymbol{l}_{m_{\alpha}}\right)=H^{\bullet}\left(B_{\rho}^{\Gamma}\right)
\end{gathered}
$$




$$
\begin{aligned}
& H^{\bullet}\left(W_{\rho}^{\Gamma}, \mathfrak{o}\left(V_{0}\right) \oplus \mathfrak{o}_{m_{-1}}(\mathbb{R}) \oplus \bigoplus_{\alpha=1}^{k} \mathfrak{u}_{m_{\alpha}}\right)=H^{\bullet}\left(X_{\rho}^{\Gamma} / S O\left(V_{0}\right) \times S O_{m_{-1}}(\mathbb{R}) \times \prod_{\alpha=1}^{k} U_{m_{\alpha}}\right) \\
& H^{\bullet}\left(W_{\rho}^{\Gamma}, O\left(V_{0}\right) \times O_{m_{-1}}(\mathbb{R}) \times \prod_{\alpha=1}^{k} U_{m_{\alpha}}\right)=H^{\bullet}\left(X_{\rho}^{\Gamma} / O\left(V_{0}\right) \times O_{m_{-1}}(\mathbb{R}) \times \prod_{\alpha=1}^{k} U_{m_{\alpha}}\right) .
\end{aligned}
$$

Proof. The proof of the first statement is similar to that of Theorem 2.10, ones uses Proposition 2.13. The second statement was essentially demonstrated in the process of proving 2.10. With Proposition 2.15, the last two statements follow by comparing the cohomologies of the Weil algebras and the topological spaces. Compare to [FU] Thm. 2.2.6.

\section{Characteristic Classes for foliations on orbifolds}

In this section, we apply the computations of the Lie algebra cohomology above to define some characteristic classes for foliations on orbifolds.

3.1. Foliations on orbifolds. Here, we introduce a notion of a foliation on an orbifold. Because all of the following constructions and computations are local, instead of general orbifolds, we will work with the global quotient, i.e. $X=M / \Gamma$, where $\Gamma$ is a finite group acting on a smooth manifold $M$. We consider a foliation $\mathcal{F}$ on $M$, which is invariant under the $\Gamma$ action, and call it a $\Gamma$ equivariant foliation on $M$. We denote by $\mathcal{F}$ both the foliation and the distribution defining the foliation.

Theorem 3.1. Let $\mathcal{F}$ be a $\Gamma$ equivariant foliation on $M$. Then for each $\gamma \in \Gamma, \mathcal{F}$ restricts to a foliation $\mathcal{F}^{\gamma}$ on $M^{\gamma}$, where $M^{\gamma}$ is the $\gamma$ fixed point manifold.

Proof. Note that $\mathcal{F}^{\gamma}$ on $M^{\gamma}$ is given by $\mathcal{F}_{x}^{\gamma}=T_{x} M^{\gamma} \cap \mathcal{F}_{x}$. We will prove that $\mathcal{F}^{\gamma}$ is indeed a foliation on $M^{\gamma}$, i.e.

(1) $\mathcal{F}^{\gamma}$ is of constant rank on each connected component of $M^{\gamma}$;

(2) $\mathcal{F}^{\gamma}$ is integrable.

We check (1) and (2) separately.

For (1), we notice that for $x \in M^{\gamma}, \gamma$ acts on $T_{x} M$ with $\mathcal{F}_{x}$ a submodule, and $\mathcal{F}_{x}^{\gamma}=\left(\mathcal{F}_{x}\right)^{G}$. Where $G=\langle\gamma\rangle$, a finite cyclic group generated by $\gamma$. Since in the representation space of $G$, the trivial representation is an isolated point ${ }^{7}$, the dimension of $\mathcal{F}_{x}^{\gamma}$ is locally constant on $M^{\gamma}$. Therefore, $\mathcal{F}^{\gamma}$ is of constant rank on each component of $M^{\gamma}$.

To check (2), we observe that a pair of sections $\eta, \nu$ of $\mathcal{F}^{\gamma}$ can locally be extended to a pair $\tilde{\eta}, \tilde{\nu}$ of $G$-invariant sections of $\mathcal{F}$. Since $\mathcal{F}$ is closed under the commutator bracket, and the $G$-action commutes with the bracket, we see that $[\tilde{\eta}, \tilde{\nu}]$ is a $G$ invariant section of $\mathcal{F}$. Thus $[\eta, \nu]=\left.[\tilde{\eta}, \tilde{\nu}]\right|_{M^{\gamma}}$ is a section of $\mathcal{F}^{\gamma}$.

\footnotetext{
${ }^{7}$ This phrasing of the proof is motivated by the remark that follows it. To prove only the statement of the theorem one can observe that the eigenvalues of $\gamma$ are $n$-th roots of unity and so cannot change continuously. This in effect proves the discreteness of the representation space.
} 
Remark 3.2. As is clear from the proof, we can extend Theorem 3.1 to the following setting. Let $G$ act on a manifold $M$ such that $M$ has a $G$-invariant hermitian structure. Suppose that $\mathcal{F}$ is a $G$-invariant foliation on $M$. Then $\mathcal{F}$ restricts to a foliation on the fixed point manifold $M^{G}$ when $G$ satisfies property $T$.

3.2. Characteristic classes. In this subsection, we want to define some characteristic classes for a foliation $\mathcal{F}$ on an orbifold $X$. As foreshadowed by Theorem 3.1, our characteristic classes map takes values in the cohomology group of the inertia orbifold $H^{\bullet}(\tilde{X})$.

We review the theory of characteristic classes for foliations on a manifold. Possible references for the standard approach to this are $[\mathrm{FU}],[\mathrm{KT}]$. Our approach differs and owes much to the point of view of $[\mathrm{K}]$. In addition we develop the equivariant version of these methods. Let $G$ be a Lie group with its Lie algebra denoted by $\mathfrak{g}$. Our discussion takes place over $\mathbb{R}$, i.e. all manifolds and Lie algebras are real. It readily modifies, where appropriate, to $\mathbb{C}$ by considering almost complex manifolds and complex Lie algebras.

Recall that a $\mathfrak{g}$-structure on a smooth manifold $M$ is a smooth 1-form $\omega$ on $M$ with values in $\mathfrak{g}$, satisfying the Maurer-Cartan equation:

$$
d \omega+\frac{1}{2}[\omega, \omega]=0 .
$$

The $\mathfrak{g}$-structure on $M$ is equivalent to the structure of a trivialized flat principal $G$-bundle $\mathcal{P}=M \times G$ over $M$. The flat connection on $\mathcal{P}$ defines a $\mathfrak{g}$-structure on $M$.

Given a $\mathfrak{g}$-structure $\omega$ on a manifold $M$, we can define a characteristic classes map $\chi_{\omega}$ by

$$
\begin{aligned}
\chi_{\omega}: \wedge^{\bullet} \mathfrak{g}^{*} & \rightarrow \Omega^{\bullet}(M) \\
\phi & \mapsto \phi \circ \omega .
\end{aligned}
$$

(Note the abuse of notation in writing $\omega$ for $\wedge^{i} \omega$.) Because $\omega$ satisfies the MaurerCartan equation, it is straightforward to check that $\chi_{\omega}$ commutes with differentials, therefore we have a map on the cohomologies:

$$
\chi_{\omega}: H^{\bullet}(\mathfrak{g}) \rightarrow H^{\bullet}(M) .
$$

The cohomology classes in the image of $\chi_{\omega}$ can be considered as characteristic classes of the $\mathfrak{g}$-structure $\omega$.

In the case of a not necessarily trivialized $\mathcal{P}$ over $M$, with the flat connection given by a $G$-equivariant $\omega \in \Omega^{1}(\mathcal{P}) \otimes \mathfrak{g}$ satisfying the Maurer-Cartan equation, we analogously obtain a map $\chi_{\omega}$

$$
\begin{aligned}
\chi_{\omega}: \wedge^{\bullet} \mathfrak{g}^{*} & \rightarrow \Omega^{\bullet}(\mathcal{P}) \\
\phi & \mapsto \phi \circ \omega .
\end{aligned}
$$

This again induces a map on cohomologies: $\chi_{\omega}: H^{\bullet}(\mathfrak{g}) \rightarrow H^{\bullet}(\mathcal{P})$. Should a trivialization of $\mathcal{P}$ exist, by choosing one, i.e. picking a section $s$ of $\mathcal{P} \rightarrow M$ we obtain a map $s^{*} \circ \chi_{\omega}: H^{\bullet}(\mathfrak{g}) \rightarrow H^{\bullet}(M)$ that recovers the above case; it depends on $s$ up to homotopy.

In order to deal with the case when a trivialization does not exist we can also consider the relative version of the above construction. Suppose that $K \subset G$ is a subgroup, denote by $p: \mathcal{P} \rightarrow \mathcal{P} / K$ the projection map. The form $\omega$ in $\Omega^{1}(\mathcal{P}) \otimes$ $\mathfrak{g}$ specifying the flat connection on $\mathcal{P}$ can be considered as a $G$-equivariant map 
$\mathfrak{g}^{*} \rightarrow \Omega_{\mathcal{P}}^{1}$. This restricts to a $K$-equivariant map $(\mathfrak{g} / \mathfrak{K})^{*} \rightarrow p^{*} \Omega_{\mathcal{P} / K}^{1}$. Thus we have $\left(\wedge^{\bullet}(\mathfrak{g} / \mathfrak{K})^{*}\right)^{K} \rightarrow\left(p^{*} \Omega_{\mathcal{P} / K}^{\bullet}\right)^{K}=\Omega_{\mathcal{P} / K}^{\bullet}$. In this way we obtain the relative version of characteristic classes:

$$
\chi_{\omega_{K}}: H^{\bullet}(\mathfrak{g}, K) \rightarrow H^{\bullet}(\mathcal{P} / K) .
$$

As before, we may pick a section $s$ of $\pi: \mathcal{P} / K \rightarrow M$ (if it exists) to get the relative characteristic classes map $s^{*} \circ \chi_{\omega_{K}}$ that again depends on the section up to homotopy. However if $G / K$ is contractible we obtain a canonical map $\left(\pi^{*}\right)^{-1} \circ \chi_{\omega_{K}}$.

In order to deal with orbifolds we must adapt the above to the equivariant setting. That is we consider a principal $G$-bundle $\mathcal{P}$ over $M$ with a $\Gamma$ action. This means that in addition to the usual structure we have the action of $\Gamma$ on $\mathcal{P}$ and $M$ that makes the projection map $\Gamma$-equivariant, and the actions of $\Gamma$ and $G$ commute, i.e. $\gamma(x g)=\gamma(x) g$ for $\gamma \in \Gamma, x \in \mathcal{P}$ and $g \in G$. If $\mathcal{P}$ is equipped with a flat connection, we require the connection to be $\Gamma$-equivariant, i.e. the connection form $\omega$ is in $\left(\Omega_{\mathcal{P}}^{1} \otimes \mathfrak{g}\right)^{\Gamma}$. We may now proceed as before with the additional observation that $\chi_{\omega_{K}}$ is $\Gamma$-equivariant and thus

$$
\chi_{\omega_{K}}: H^{\bullet}(\mathfrak{g}, K) \rightarrow H^{\bullet}(\mathcal{P} / K)^{\Gamma}
$$

and in the case that $G / K$ is contractible the image of $\left(\pi^{*}\right)^{-1} \circ \chi_{\omega_{K}}$ is contained in $H^{\bullet}(M)^{\Gamma}$.

Consider a $\gamma \in \Gamma$ and let $M^{\gamma}=\amalg M_{i}^{\gamma}$, where $M_{i}^{\gamma}$ is a $\Gamma^{\gamma}$-orbit of a connected component of $M^{\gamma}$. To any $x \in M_{i}^{\gamma}$ we may associate $\left\langle\gamma_{x}\right\rangle$ a conjugacy class in $G$ of $\gamma_{x} \in G$ that satisfies $y \gamma_{x}=\gamma(y)$ for some choice of $\left.y \in \mathcal{P}\right|_{x}$. While $\gamma_{x}$ depends on $y$, its conjugacy class in $G$ does not. If $\mathcal{P}$ is equipped with a $\Gamma$-equivariant flat connection then $\left\langle\gamma_{x}\right\rangle$ will be constant on each connected component of $M_{i}^{\gamma}$ and thus on $M_{i}^{\gamma}$ itself. Thus for a choice of $\gamma_{i} \in\left\langle\gamma_{x}\right\rangle$ (notice that $\gamma_{i} \in G$ unlike $\gamma \in \Gamma$ ) we may reduce the structure of $\left.\mathcal{P}\right|_{M_{i}^{\gamma}}$ to $G^{\gamma_{i}}$. Explicitly, we consider the principal $G^{\gamma_{i}}$-bundle with the fiber over $x \in M_{i}^{\gamma}$ consisting of all $\left.y \in \mathcal{P}\right|_{x}$ such that $\gamma(y)=y \gamma_{i}$. Let us denote this principal $G^{\gamma_{i}}$-bundle over $M_{i}^{\gamma}$ by $\mathcal{P}^{\gamma_{i}}$. It is evidently $\Gamma^{\gamma}$-equivariant and it is easy to see that the $\Gamma$-equivariant connection on $\mathcal{P}$ will induce a $\Gamma^{\gamma}$-equivariant connection on $\mathcal{P}^{\gamma_{i}}$.

The characteristic classes formalism above can then be used to supply the classes associated with $\mathcal{P}$. Namely, for every $\gamma \in \Gamma$ (we need only choose one per conjugacy class), and a choice of $M_{i}^{\gamma} \subset M^{\gamma}$, choose a $\gamma_{i} \in\left\langle\gamma_{x}\right\rangle \subset G$ and suppose further that we have a $K_{\gamma_{i}} \subset G^{\gamma_{i}}$ with $G^{\gamma_{i}} / K_{\gamma_{i}}$ contractible. Then we get a characteristic classes map

$$
H^{\bullet}\left(\mathfrak{g}^{\gamma_{i}}, K_{\gamma_{i}}\right) \rightarrow H^{\bullet}\left(M_{i}^{\gamma}\right)^{\Gamma^{\gamma}}
$$

that essentially does not depend on the choices of conjugacy class representatives. We note that the classes obtained in this way are located inside the cohomology of the inertia orbifold $\widetilde{X}$.

Now we can apply all this to obtain characteristic classes of foliations. The key to the application is the consideration of an appropriate principal $G$-bundle with flat connection. The "group" $G$ will be the cross product of the group of formal coordinates around $0 \in \mathbb{R}^{n}$ and the group of formal translations in $\mathbb{R}^{n}$. Denote this group by $\operatorname{Dif} f_{n}$ and note that its Lie algebra is $W_{n}$. Observe that $G L_{n}(\mathbb{R}) \subset$ Diff $f_{n}$ and $\operatorname{Diff}_{n} / G L_{n}(\mathbb{R})$ is contractible. 
First, let us recall the non-equivariant situation. Let $\mathcal{F}$ be a foliation on a manifold $M$ of codimension $n$. We define the principal Dif $f_{n}$-bundle $\mathcal{P}_{\text {Diff }}$ over $M$ as follows. Let $\hat{\mathbb{R}}_{0}^{n}$ denote the formal neighborhood of 0 in $\mathbb{R}^{n}$ and $A u t_{n}$ the group of diffeomorphisms of $\hat{\mathbb{R}}_{0}^{n}$. With this notation Diff $f_{n}=A u t_{n} \ltimes \hat{\mathbb{R}}_{0}^{n}$. Define the $A u t_{n^{-}}$ bundle $\mathcal{P}_{\text {Aut }}$ by setting the fiber over $x \in M$ to be the set of diffeomorphisms from $\hat{\mathbb{R}}_{0}^{n}$ to the formal neighborhood of $x$ in the local leaf space around $x$, i.e. the quotient of the formal neighborhood of $x \in M$ by the connected components of the leaves. Let $\mathcal{P}_{\text {Diff }}=\mathcal{P}_{\text {Aut }} \times_{\text {Aut }_{n}}$ Diff $f_{n}$. The resulting Diff $f_{n}$-bundle has a canonical flat connection. By the general theory we get a map:

$$
H^{\bullet}\left(W_{n}, G L_{n}(\mathbb{R})\right) \rightarrow H^{\bullet}(M)
$$

that encodes information about the foliation $\mathcal{F}$. More precisely, these are the real Pontryagin classes of the normal bundle $N \mathcal{F}$ to $\mathcal{F}$ (see Remark 2.7). As pointed out in $[\mathrm{FU}]$ this gives the well known vanishing theorem of Bott: monomials of the real Pontryagin classes of $N \mathcal{F}$ that have degree $>2 n$ are equal to 0 . However one does obtain extra information using this method, namely since $G L_{n}(\mathbb{R}) / O_{n}(\mathbb{R})$ is contractible we have:

$$
H^{\bullet}\left(W_{n}, O_{n}(\mathbb{R})\right) \rightarrow H^{\bullet}(M)
$$

that extends the map above and thus defines extra classes called secondary characteristic classes for a general foliation.

We can refine this type of analysis in the special case of a framed foliation. Suppose that $N \mathcal{F}$ is trivialized. Choosing a metric yields an exponential map that gives a reduction of structure of $\mathcal{P}_{\text {Diff }}$ to $F(N \mathcal{F})$, the frame bundle of $N \mathcal{F}$. At the same time the trivialization provides a smooth section of $F(N \mathcal{F})$ and since any two metrics are homotopic, we have a canonical map:

$$
H^{\bullet}\left(W_{n}\right) \rightarrow H^{\bullet}(M)
$$

encoding the data of the framing as well.

Now we want to use the above idea to define characteristic classes for a foliation on an orbifold. For simplicity of exposition let us assume that we are in the setting of a manifold $M$ with a finite group $\Gamma$ action that preserves a foliation $\mathcal{F}$ (of codimension $n$ ) on $M$. Then the principal Dif $f_{n}$-bundle $\mathcal{P}_{\text {Diff }}$ as defined above is automatically $\Gamma$-equivariant with an equivariant flat connection. One can choose a $\Gamma$-equivariant metric on $N \mathcal{F}$ (any two such metrics are homotopic), then the exponential map gives a $\Gamma$-equivariant reduction of structure of $\mathcal{P}_{D i f f}$ to $F(N \mathcal{F})$. This reduction allows us to choose $\gamma_{i} \in G L_{n}(\mathbb{R})$. Since $\operatorname{Diff}_{n}^{\gamma_{i}} / G L_{n}^{\gamma_{i}}(\mathbb{R})$ is contractible, we have the characteristic classes map:

$$
H^{\bullet}\left(W_{n}^{\gamma_{i}}, G L_{n}^{\gamma_{i}}(\mathbb{R})\right) \rightarrow H^{\bullet}\left(M_{i}^{\gamma}\right)^{\Gamma^{\gamma}}
$$

that can, similarly to the non-equivariant discussion, be interpreted as a mixture of Pontryagin and Chern classes for the direct sum decomposition of the restriction of $N \mathcal{F}$ to $M_{i}^{\gamma}$ induced by the action of $\gamma$. Namely, by restricting $N \mathcal{F}$ to $M_{i}^{\gamma}$ we obatin a real vector bundle with a fiberwise action of $\gamma$. This action produces a decomposition into irreducible component subbundles. The components on which the action is diagonalizable give rise to the Pontryagin classes, whereas the remaining part of the decomposition acquires a complex structure and so defines Chern classes. Consequently we obtain an equivariant version of Bott's vanishing theorem that is 
stronger than the original. More precisely, monomials in the Pontryagin and Chern classes having degree exceeding $2 \operatorname{rank}\left(N \mathcal{F}^{\gamma}\right)$ are cohomologically trivial(see Lemma 2.6 and Remark 2.7).

To obtain extra information beyond the standard characteristic classes, observe that $G L_{n}^{\gamma_{i}}(\mathbb{R}) / O_{n}^{\gamma_{i}}(\mathbb{R})$ is contractible, thus we have:

$$
\chi_{\gamma, i}: H^{\bullet}\left(W_{n}^{\gamma_{i}}, O_{n}^{\gamma_{i}}(\mathbb{R})\right) \rightarrow H^{\bullet}\left(M_{i}^{\gamma}\right)^{\Gamma^{\gamma}} .
$$

Explicitly, using the conventions of Section 2.4, the action of $\gamma_{i}$ on $\mathbb{R}^{n}$ gives a decomposition $\mathbb{R}^{n}=V_{0} \oplus m_{-1} W_{-1} \oplus \bigoplus_{\alpha=1}^{k} m_{\alpha} W_{\alpha}$. Then $G L_{n}^{\gamma_{i}}(\mathbb{R})=G L\left(V_{0}\right) \times G L_{m_{-1}}(\mathbb{R}) \times$ $\prod G L_{m_{\alpha}}(\mathbb{C})$ and $O_{n}^{\gamma_{i}}(\mathbb{R})=O\left(V_{0}\right) \times O_{m_{-1}}(\mathbb{R}) \times \prod U_{m_{\alpha}}$.

Definition 3.3. For a foliation $\mathcal{F}$ on an orbifold $X$, we define the characteristic classes of $\mathcal{F}$ to be the elements in the image of the map

$$
\chi=\oplus \chi_{\gamma, i}: \bigoplus_{\langle\gamma\rangle \subset \Gamma, i} H^{\bullet}\left(W_{n}^{\gamma_{i}}, O_{n}^{\gamma_{i}}(\mathbb{R})\right) \rightarrow H^{\bullet}(\tilde{X}) .
$$

The cohomology of $H^{\bullet}\left(W_{n}^{\gamma_{i}}, O_{n}^{\gamma_{i}}(\mathbb{R})\right)$ is computed in Theorem 2.18. It is isomorphic to the cohomology of the topological space $X_{\rho}^{\gamma_{i}} / O_{n}^{\gamma_{i}}$, which is fibered over $B_{\rho}^{\gamma_{i}}$. The cohomology ring of $B_{\rho}^{\gamma_{i}}$ is the polynomial ring with $\operatorname{dim}\left(V_{0}\right)+m_{-1}+2 \sum m_{\alpha}$ generators truncated at degree $>2 \operatorname{dim}\left(V_{0}\right)$. The images under $\chi$ of these generators correspond to the Pontryagin and Chern classes as explained above. Furthermore, we notice that $X_{\rho}^{\gamma_{i}} / O_{n}^{\gamma_{i}} \rightarrow B_{\rho}^{\gamma_{i}}$ has a cohomologically nontrivial fiber $U_{\operatorname{dim}\left(V_{0}\right)} \times U_{m_{-1}} \times \prod\left(U_{m_{\alpha}} \times U_{m_{\alpha}}\right) / O\left(V_{0}\right) \times O_{m_{-1}}(\mathbb{R}) \times \prod U_{m_{\alpha}}$. Thus the cohomology group of $X_{\rho}^{\gamma_{i}} / O_{n}^{\gamma_{i}}$ is larger than the one of $B_{\rho}^{\gamma_{i}}$. The extra cohomology classes give rise to the secondary characteristic classes of $\mathcal{F}$. For example, looking at the $E_{2}$ term of the spectral sequence associated to the fibration, we see that any class in the upper right hand corner will survive as a class in the cohomology of $X_{\rho}^{\gamma_{i}} / O_{n}^{\gamma_{i}}$. These can be thought of as generalizations of the Godbillon-Vey class.

Example 1. We consider the 0-dimensional foliation $\mathcal{F}$ on a manifold $M$. Any finite group $\Gamma$ action on this manifold preserves $\mathcal{F}$. Definition 3.3 defines on each piece of $M^{\gamma}$ characteristic classes of the tangent bundle $T M^{\gamma}$, and also characteristic classes of the normal bundle $N M^{\gamma}$ of the embedding $M^{\gamma} \hookrightarrow M$. Correspondingly, on the inertia orbifold $\tilde{X}$, Definition 3.3 defines characteristic classes of both the tangent bundle of each $X^{\gamma}$ and also the normal bundle of $X^{\gamma} \hookrightarrow X$. These provide all the characteristic classes needed in the topological index of an elliptic differential operator on an orbifold [PPT].

Example 2. Let $\mathcal{F}$ be a foliation on a manifold $M$. We consider the manifold $M \times M$ with the foliation $\mathcal{F} \times \mathcal{F}$. We have the group $\mathbb{Z}_{2}=\mathbb{Z} / 2 \mathbb{Z}$ acting on $M \times M$ by switching the components. This action preserves the foliation $\mathcal{F} \times \mathcal{F}$. We write elements in $\mathbb{Z}_{2}$ as $i d$ and $\sigma$. Associated to the element $i d$, Definition 3.3 defines Gelfand-Fuchs classes of the foliation $\mathcal{F} \times \mathcal{F}$ on $M \times M$, which are invariant under $\mathbb{Z}_{2}$ action. For the element $\sigma$, its fixed point set is the diagonal $\Delta$ in $M \times M$, which is diffeomorphic to $M$. The intersection $\mathcal{J}$ of the foliation $\mathcal{F} \times \mathcal{F}$ with the diagonal $\Delta$ defines a foliation on $\Delta$, which is equal to $\mathcal{F}$ if we identify $\Delta$ with $M$. We denote the restriction to $\Delta$ of the normal bundle of $\mathcal{F} \times \mathcal{F}$ inside $T M \times T M$ by $N \mathcal{F}$. $\sigma$ acts on 
$N \mathcal{F}$ fiberwisely, and we denote the corresponding -1 eigen subbundle in $N \mathcal{F}$ by $N \mathcal{J}$. Then associated to the element $\sigma$, Definition 3.3 defines Gelfand-Fuchs classes of $\mathcal{J}$ on $\Delta$, and also the characteristic classes of $N \mathcal{J}$ on $\Delta$. Under the identification $(\Delta, \mathcal{J})$ with $(M, \mathcal{F})$, the Gelfand-Fuchs classes of $\mathcal{J}$ on $\Delta$ and $\mathcal{F}$ on $M$ are indentified. We see that the $\sigma$ component of the Definition 3.3 contains the information of the original foliation $(M, \mathcal{F})$. We remark that one easily generalizes this example to $M^{\times n}$ and the $n$-th permutation group $\Sigma_{n}$. Definition 3.3 provides much more information than the Gelfand-Fuchs classes of the foliation $\mathcal{F}^{\times n}$ on $M^{\times n}$.

Remark 3.4. Different approaches such as [KT] can also be used to define characteristic classes for foliations on orbifolds. Here, the Gelfand-Fuchs cohomology approach is used as an application of the Lie algebra cohomology computation.

\section{Acknowledgements.}

Both authors are indebted to D.B. Fuchs for encouragements and advice, as well as J. Kaminker, M. Pflaum, and H. Posthuma for useful conversations. Research of the second author was supported in part by NSF Grant 0604552.

\section{References}

[BW] A. Borel and N. Wallach, Continuous cohomology, discrete subgroups, and representations of reductive groups, Second edition, Mathematical Surveys and Monographs, 67. American Mathematical Society, Providence, RI, 2000.

[C] A. Connes, Noncommutative geometry, Academic Press, Inc., San Diego, CA, 1994.

[CM] A. Connes and H. Moscovici, Hopf algebras, cyclic cohomology and the transverse index theorem, Comm. Math. Phys. 198 (1998), no. 1, 199-246.

[FFS] B. Feigin, G. Felder, and B. Shoikhet, Hochschild cohomology of the Weyl algebra and traces in deformation quantization, Duke Math. J. 127 (2005), no. 3, 487-517.

[Fu] D. Fuchs Cohomology of Infinite dimensional Lie algebras, Contemp. Soviet Math., Consultants Bureau, New York, 1986.

[KT] F. Kamber and P. Tondeur, Foliated bundles and characteristic classes, Lecture Notes in Mathematics, Vol. 493. Springer-Verlag, Berlin-New York, 1975.

[K] M. Kontsevich, Rozansky-Witten invariants via formal geometry, Compositio Math. 115 (1999), no. $1,115-127$.

[NT] R. Nest and B. Tsygan, Algebraic index theorem, Comm. Math. Phys. 172 (1995), no. 2, 223-262.

[PPT] M. Pflaum, H. Posthuma, and X. Tang, An algebraic index theorem for orbifolds, Adv. Math. 210 (2007), no. 1, 83-121.

Max Planck Institute for Mathematics, Vivatsgasse 7, 53111 Bonn Germany

E-mail address: ishapiro@mpim-bonn.mpg.de

Department of Mathematics, Washington University, St. Louis, MO, 63130, USA

E-mail address: xtang@math.wustl.edu 